\title{
Parametric and Internal Resonances of an Axially Moving Beam with Time-Dependent Velocity
}

\author{
Bamadev Sahoo, ${ }^{1}$ L. N. Panda, ${ }^{2}$ and G. Pohit ${ }^{3}$ \\ ${ }^{1}$ Department of Mechanical Engineering, International Institute of Information Technology, Bhubaneswar 751003, India \\ ${ }^{2}$ Department of Mechanical Engineering, College of Engineering and Technology, Bhubaneswar 751003, India \\ ${ }^{3}$ Department of Mechanical Engineering, Jadavpur University, Kolkata 700032, India
}

Correspondence should be addressed to G. Pohit; gpohit@gmail.com

Received 10 May 2013; Accepted 27 August 2013

Academic Editor: Abdelali El Aroudi

Copyright (C) 2013 Bamadev Sahoo et al. This is an open access article distributed under the Creative Commons Attribution License, which permits unrestricted use, distribution, and reproduction in any medium, provided the original work is properly cited.

\begin{abstract}
The nonlinear vibration of a travelling beam subjected to principal parametric resonance in presence of internal resonance is investigated. The beam velocity is assumed to be comprised of a constant mean value along with a harmonically varying component. The stretching of neutral axis introduces geometric cubic nonlinearity in the equation of motion of the beam. The natural frequency of second mode is approximately three times that of first mode; a three-to-one internal resonance is possible. The method of multiple scales (MMS) is directly applied to the governing nonlinear equations and the associated boundary conditions. The nonlinear steady state response along with the stability and bifurcation of the beam is investigated. The system exhibits pitchfork, Hopf, and saddle node bifurcations under different control parameters. The dynamic solutions in the periodic, quasiperiodic, and chaotic forms are captured with the help of time history, phase portraits, and Poincare maps showing the influence of internal resonance.
\end{abstract}

\section{Introduction}

Band saws, fibre textiles, magnetic tapes, paper sheets, aerial tramways, pipes transporting fluids, thread lines, and belts are some technological examples classified as axially moving continua. Analytical models for axially moving systems have been extensively used in the last few decades. The vast literature on axially moving continua vibration has been reviewed by Wickert and Mote Jr. [1] up to 1988. While a linear analysis provides natural frequencies, mode shapes, and critical speeds, its validity regarding the response of the system diminishes as the vibration amplitude becomes sufficiently large or as the critical speed is approached [2]. In these cases one must resort to a nonlinear analysis. Wickert and Mote Jr. [3,4] studied the transverse vibration of axially moving strings and beams using an eigenfunction method. They also studied the dynamic response of an axially moving string loaded suspended mass. Wickert [5] presented a detailed study of the nonlinear vibrations and bifurcations of moving beams using the Krylov-Bogoliubov-Mitropolsky asymptotic method. Chakraborty et al. $[6,7]$ investigated both free and forced vibration of the nonlinear traveling beam using complex normal modes.

There are papers devoted to the analysis of the dynamic behavior of traveling systems with time-dependent axial velocity or with time-dependent axial tension force. Öz and Pakdemirli [8] investigated principal parametric resonances and combination resonances of sum and difference types for any two modes for an axially accelerating beam using the method of multiple scales. They found that for combination resonances, instabilities occurred only for additive type but not for difference type. Öz et al. [9] extended the work to nonlinear transverse vibration and stability analysis. Comprehensive review of nonlinear modal interactions is there in [10-12]. Using method of multiple scales Riedel and Tan [13] studied the coupled and forced behavior of an axially moving strip with internal resonance. Özkaya et al. [14] investigated nonlinear transverse vibrations and $3: 1$ internal resonances of a beam with multiple supports and plotted frequency response curves for different support numbers. Bagdatli et al. [15] extended this work to find existence of internal resonance cases between different modes. Chin and 
Nayfeh [16] investigated three-to-one internal resonances in parametrically excited hinged-clamped beams. They studied principal parametric resonance of the first or the second mode and a combination parametric resonance of additive type of these modes. Panda and $\operatorname{Kar}[17,18]$ investigated the principal parametric resonance of first mode, second mode and combination parametric resonance in presence of 3:1 internal resonance of a pipe conveying pulsating fluid with pinned-pinned end conditions. They studied the equilibria of these modulated equations and determined their stability, and then they did extensive dynamic study at typical initial conditions. Sze et al. [19] used incremental harmonic balance method for nonlinear vibration of axially moving beams. They investigated the fundamental, superharmonic, and subharmonic resonance in presence of internal resonance. Huang et al. [20] used the same method to analyse the stability and bifurcation analysis of a traveling beam tuned to $3: 1$ internal resonance with attention to fundamental and subharmonic resonances. Chen et al. [21] investigated dynamic stability of an axially accelerating viscoelastic beam undergoing parametric resonance. Ding et al. [22, 23] used Galerkin methods for finding natural frequencies of high-speed axially moving beams with hybrid boundary conditions. Marynowski and Kapitaniak [24-26] introduced several internal dissipation mechanisms like Kelvin-voigt and Zener in the modeling traveling continua. Pakdemirli and Öz [27] studied the transverse vibration of simply supported axially moving Euler-Bernoulli beam for infinite mode analysis and truncation to resonant modes. Ponomareva and van Horssen [28] investigated transversal vibrations of axially travelling continua based on a string model at the low frequencies and a tensioned beam model at the higher frequencies. Recently, a systematic research on travelling beam was pursued by Ghayesh et al. [29-33] involving nonlinear dynamic phenomenon of a variety of system models. The forced dynamics of an axially moving viscoelastic beam was investigated in [29]. The nonlinear vibrations and stability of an axially moving beam subjected to a distributed harmonic excitation load were investigated in [30]. In addition, the response of the system tuned to a three-to-one internal resonance was also examined. Subcritical dynamics of the system was examined [31] via the pseudoarclength continuation technique, while the global dynamics was investigated using direct time integration.

The present work aims to investigate the problem of a simply supported traveling beam under parametric excitation due to the harmonic pulsation in the travelling velocity. For a certain range of mean velocity, the natural frequency of the second mode is approximately three times that of the first mode. This relationship between natural frequencies of first two modes of a system having cubic nonlinearity results in a condition of three-to-one autoparametric or internal resonance leading to energy exchange between the two involved modes through nonlinear modal interaction. In the present work, the principal parametric resonance of the first mode is considered. The system behavior shows pitchfork, Hopf, and saddle node bifurcations in steady state analysis. Decreasing internal frequency detuning parameter affects the amplitude of directly excited first mode and the number of

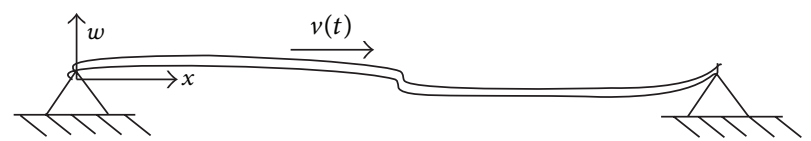

FIgURE 1: Schematic diagram of an axially traveling simply supported beam with variable velocity.

Hopf bifurcation points and shifts the occurrence of jump phenomena. Due to the influence of internal resonance, the dynamic behavior of the system exhibits periodic, quasiperiodic, and chaotic responses. The results are presented in the form of time histories, phase plane portraits, fast Fourier transforms (FFTs), and Poincare maps.

\section{Formulation of the Problem}

For the present work, a uniform horizontal beam simply supported at both ends and travelling with a harmonically variable velocity (Figure 1) is considered. The assumptions taken here are (1) the motion of the beam is planar, (2) the uniform cross sections remain plane during the motion and the beam behaves like an Euler-Bernoulli beam in transverse vibration, and (3) the type of nonlinearity is geometric due to the midplane stretching effect of the beam. The nondimensional equation of transverse motion of the beam including the nonlinearity due to midplane stretching $[5,9]$ and viscous damping [34], along with viscoelastic damping [35], is given by

$$
\begin{aligned}
\ddot{w} & +2 v \dot{w}^{\prime}+\dot{v} w^{\prime}+\left(v^{2}-1\right) w^{\prime \prime}+v_{f}^{2} w^{\prime \prime \prime \prime} \\
& +2 \varepsilon \alpha \dot{w}^{\prime \prime \prime \prime}+2 \varepsilon \mu \dot{w}=\frac{1}{2} v_{l}^{2} w^{\prime \prime} \int_{0}^{1} w^{\prime 2} d x .
\end{aligned}
$$

The nondimensional scheme used here is

$$
\begin{gathered}
x=\frac{x^{*}}{L}, \quad t=t^{*} \sqrt{\frac{P}{\rho A L^{2}}}, \quad w=\frac{w^{*}}{L}, \\
v=\frac{v^{*}}{\sqrt{P / \rho A}}, \quad 2 \varepsilon \alpha=\frac{E^{*}}{L^{2}}\left(\frac{I}{m E}\right)^{1 / 2}, \\
2 \varepsilon \mu=\frac{C L^{2}}{\sqrt{m E I}}, \quad v_{l}=\sqrt{\frac{E A}{P}}, \quad v_{f}=\sqrt{\frac{E I}{P L^{2}}},
\end{gathered}
$$

where the variables with asterisk denote dimensional ones. In the EOM, dot denotes derivatives with respect to time $(t)$ and the prime denotes derivatives with respect to spatial derivative $(x) . m$ is mass per unit length, $\rho$ is density, $A$ is cross-sectional area, $L$ is length, $v_{f}$ is nondimensional flexural stiffness, and $v_{l}$ is nondimensional longitudinal stiffness of beam. $E^{*}$ is the coefficient of internal dissipation of the beam material, which is assumed to be Kelvin-Voigt type viscoelastic, and $C$ is the external damping factor. $\alpha$ is nondimensional material damping, and $\mu$ is nondimensional viscous damping. Reordering the transverse displacement with the relation, $w=\sqrt{\varepsilon} w^{\#}$, where $\varepsilon<1$, and putting it in the equation of motion (1), the system is converted into a weakly nonlinear one [9]. 


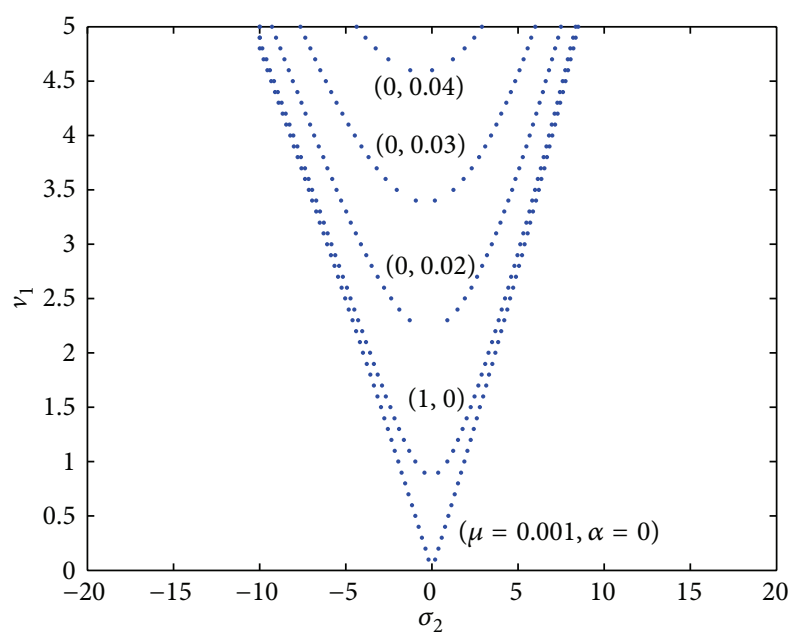

FIgURE 2: Trivial state boundary for different damping parameters. Values of the nondimensional damping parameters $(\mu, \alpha)$, indicated on the curves.

For convenience, the superscript "\#” is removed, and the weakly nonlinear equation of motion becomes

$$
\begin{aligned}
\ddot{w} & +2 v \dot{w}^{\prime}+\dot{v} w^{\prime}+\left(v^{2}-1\right) w^{\prime \prime}+v_{f}^{2} w^{\prime \prime \prime \prime} \\
& +2 \varepsilon \alpha \dot{w}^{\prime \prime \prime \prime}+2 \varepsilon \mu \dot{w}=\frac{1}{2} \varepsilon v_{l}^{2} w^{\prime \prime} \int_{0}^{1} w^{\prime 2} d x,
\end{aligned}
$$

The variable velocity of the beam is

$$
v=v_{0}+\varepsilon v_{1} \sin \Omega t,
$$

where $v_{0}$ is mean velocity, $\varepsilon v_{1}$ is the amplitude, and $\Omega$ is the frequency of the harmonically varying component. This harmonic component of velocity, which is the parametric excitation term in the equation of motion, gives various parametric responses of the system. Putting (4) in (3), we get the equation of transverse motion

$$
\begin{aligned}
2 \varepsilon \alpha \dot{w}^{\prime \prime \prime \prime} & +v_{f}^{2} w^{\prime \prime \prime \prime}+\left[v_{0}^{2}+2 \varepsilon v_{0} v_{1} \sin \Omega t-1\right] w^{\prime \prime} \\
& +\varepsilon v_{1} \Omega w^{\prime} \cos \Omega t+2\left(v_{0}+\varepsilon v_{1} \sin \Omega t\right) \dot{w}^{\prime} \\
& +2 \varepsilon \mu \dot{w}+\ddot{w}=\frac{1}{2} \varepsilon v_{l}^{2} w^{\prime \prime} \int_{0}^{1} w^{\prime 2} d x
\end{aligned}
$$

with boundary conditions

$$
w(0, t)=w(1, t)=w^{\prime \prime}(0, t)=w^{\prime \prime}(1, t)=0,
$$

The linear natural frequencies for various modes vary with variation in the mean velocity of beam. For a considerable range of mean velocity, the first two natural frequencies become commensurable, leading to modal interactions through internal resonance. The combination of internal resonance with different kinds of parametric resonances gives the system response in the form of directly excited mode and indirectly excited one because of the energy exchange between them [16-18].

\section{Method of Analysis}

An approximate solution to this weakly nonlinear distributed parameter system in the form of a first order uniform expansion by using the direct perturbation technique of method of multiple scales (MMS) [14-18] is aimed. The time scale used here is $T_{n}=\varepsilon^{n} t, n=0,1,2,3 \ldots$, and the time derivatives are

$$
\begin{aligned}
& \frac{d}{d t}=D_{0}+\varepsilon D_{1}+\cdots, \quad \frac{d^{2}}{d t^{2}}=D_{0}^{2}+2 \varepsilon D_{0} D_{1}+\cdots, \\
& D_{n}=\frac{\partial}{\partial T_{n}}, \quad n=0,1,2,3 \ldots
\end{aligned}
$$

Assuming an expansion of the form

$$
w(x, t, \varepsilon)=w_{0}\left(x, T_{0}, T_{1}\right)+\varepsilon w_{1}\left(x, T_{0}, T_{1}\right)+\cdots .
$$

Substituting (7) and (8) into (5) and (6) and equating coefficients of like powers of $\varepsilon$ on both sides, we get

$$
\begin{gathered}
O\left(\varepsilon^{0}\right): D_{0}^{2} w_{0}+2 v_{0} D_{0} w_{0}^{\prime}+\left(v_{0}^{2}-1\right) w_{0}^{\prime \prime}+v_{f}^{2} w_{0}^{\prime \prime \prime \prime}=0, \\
w_{0}(0, t)=w_{0}(1, t)=w_{0}^{\prime \prime}(0, t)=w_{0}^{\prime \prime}(1, t)=0, \\
O\left(\varepsilon^{1}\right): D_{0}^{2} w_{1}+2 v_{0} D_{0} w_{1}^{\prime}+v_{f}^{2} w_{1}^{\prime \prime \prime \prime}+\left(v_{0}^{2}-1\right) w_{1}^{\prime \prime} \\
=-2 v_{0} D_{1} w_{0}^{\prime}-2 v_{1} \sin \Omega t D_{0} w_{0}^{\prime}-2 D_{0} D_{1} w_{0} \\
-2 \alpha D_{0} w_{0}^{\prime \prime \prime \prime}-2 \mu D_{0} w_{0}-2 v_{0} v_{1} \sin \Omega t w_{0}^{\prime \prime} \\
-v_{1} \Omega \cos \Omega t w_{0}^{\prime}+\frac{1}{2} v_{l}^{2} w_{0}^{\prime \prime} \int_{0}^{1} w_{0}^{\prime 2} d x=0, \\
w_{1}(0, t)=w_{1}(1, t)=w_{1}^{\prime \prime}(0, t)=w_{1}^{\prime \prime}(1, t)=0 .
\end{gathered}
$$

The solution of (9) may be written as

$$
w_{0}\left(T_{0}, T_{1}, x\right)=\sum_{n=1}^{\infty} \phi_{n}(x) A_{n}\left(T_{1}\right) e^{i \omega_{n} T_{0}}+c c
$$

where $\phi_{n}$ is the mode shapes, $\omega_{n}$ is the natural frequencies, and $c c$ is complex conjugate. The mode shapes are calculated previously [8] as

$$
\begin{aligned}
\phi_{n}(x)=C_{1 n}\left\{e^{i \beta_{1 n} x}-\frac{\left(\beta_{4 n}^{2}-\beta_{1 n}^{2}\right)\left(e^{i \beta_{3 n}}-e^{i \beta_{1 n}}\right)}{\left(\beta_{4 n}^{2}-\beta_{2 n}^{2}\right)\left(e^{i \beta_{3 n}}-e^{i \beta_{2 n}}\right)} e^{i \beta_{2 n} x}\right. \\
-\frac{\left(\beta_{4 n}^{2}-\beta_{1 n}^{2}\right)\left(e^{i \beta_{2 n}}-e^{i \beta_{1 n}}\right)}{\left(\beta_{4 n}^{2}-\beta_{3 n}^{2}\right)\left(e^{i \beta_{2 n}}-e^{i \beta_{3 n}}\right)} e^{i \beta_{3 n} x} \\
+\left[-1+\frac{\left(\beta_{4 n}^{2}-\beta_{1 n}^{2}\right)\left(e^{i \beta_{3 n}}-e^{i \beta_{1 n}}\right)}{\left(\beta_{4 n}^{2}-\beta_{2 n}^{2}\right)\left(e^{i \beta_{3 n}}-e^{i \beta_{2 n}}\right)}\right. \\
\left.\left.+\frac{\left(\beta_{4 n}^{2}-\beta_{1 n}^{2}\right)\left(e^{i \beta_{2 n}}-e^{i \beta_{1 n}}\right)}{\left(\beta_{4 n}^{2}-\beta_{3 n}^{2}\right)\left(e^{i \beta_{2 n}}-e^{i \beta_{3 n}}\right)} e^{i \beta_{4 n} x}\right]\right\},
\end{aligned}
$$




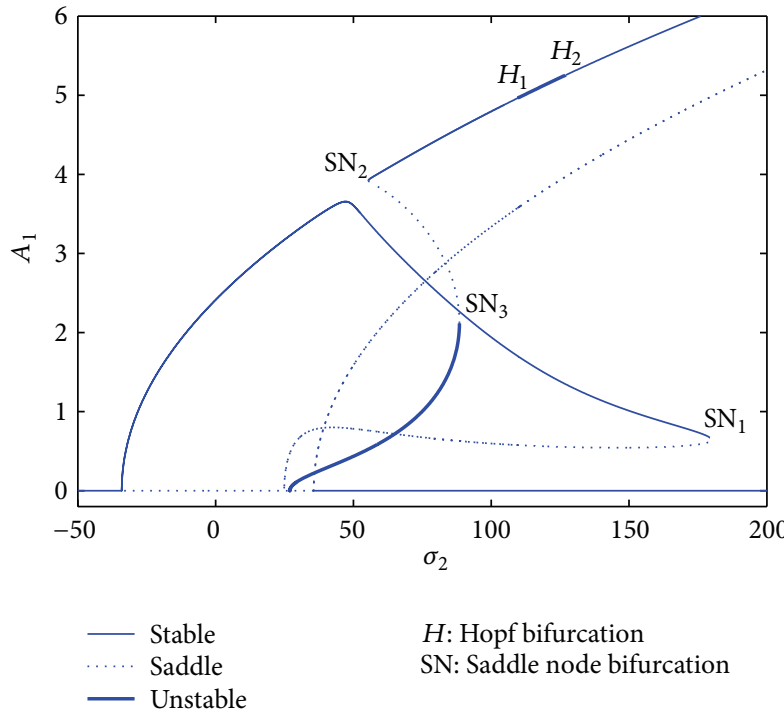

(a)

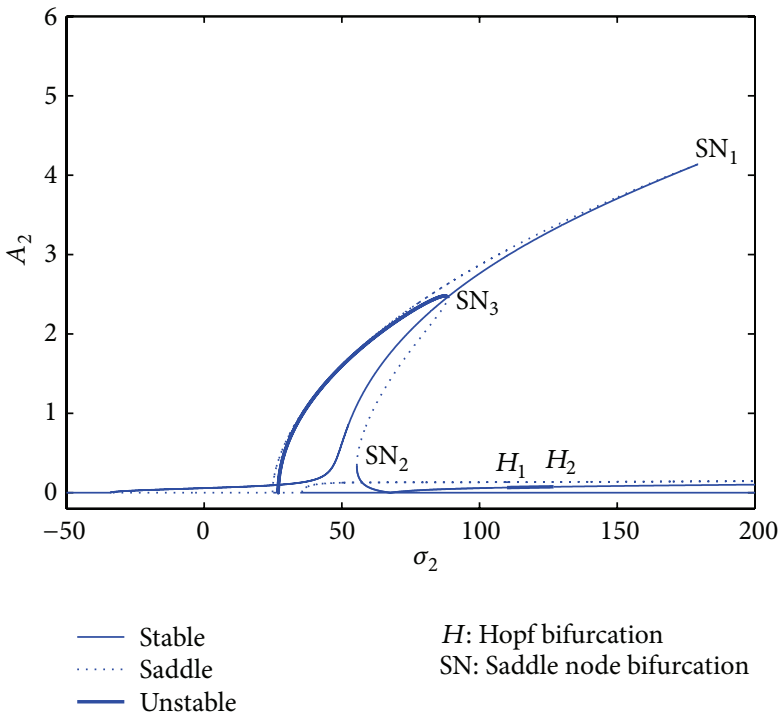

(b)

FIGURE 3: Frequency response curves as obtained by continuation algorithm for the first and second modes when the first mode is parametrically excited for the system parameters, Chin and Nayfeh [16].

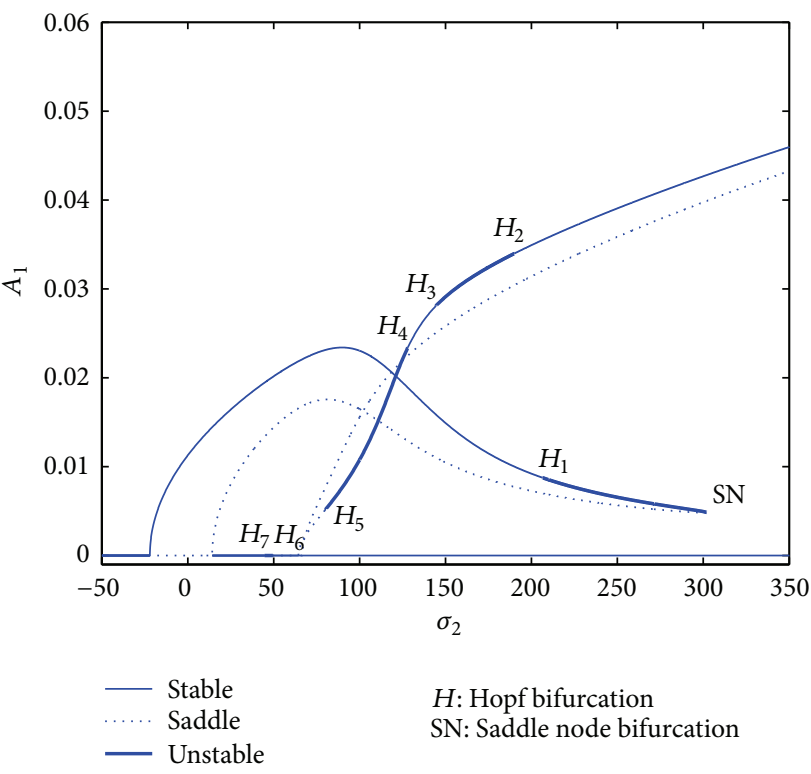

(a)

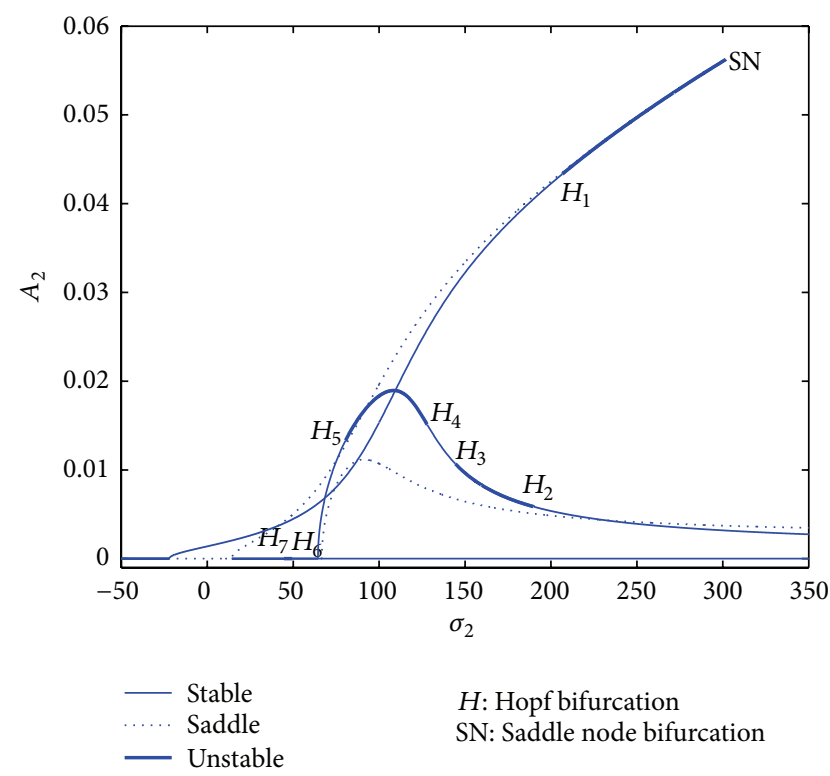

(b)

FIGURE 4: Frequency response curves for the (a) first mode and (b) second mode when the first mode is parametrically excited for the system parameters $\mu=0.1, \alpha=0, v_{1}=10, v_{l}=40$, and $\sigma_{1}=92.39$.

where $\beta_{\text {in }}$ are eigenvalues which satisfy the dispersive relation (13) and support condition (14) [8]:

$$
\begin{gathered}
v_{f}^{2} \beta_{\mathrm{in}}^{4}-\left(v_{0}^{2}-1\right) \beta_{\mathrm{in}}^{2}-2 v_{0} \omega_{n} \beta_{\mathrm{in}}-\omega_{n}^{2}=0, \\
i=1,2,3,4, \\
\left(e^{i\left(\beta_{1 n}+\beta_{2 n}\right)}+e^{i\left(\beta_{3 n}+\beta_{4 n}\right)}\right)\left(\beta_{1 n}^{2}-\beta_{2 n}^{2}\right)\left(\beta_{3 n}^{2}-\beta_{4 n}^{2}\right) \\
+\left(e^{i\left(\beta_{1 n}+\beta_{3 n}\right)}+e^{i\left(\beta_{2 n}+\beta_{4 n}\right)}\right)\left(\beta_{2 n}^{2}-\beta_{4 n}^{2}\right)
\end{gathered}
$$

$$
\begin{aligned}
& \times\left(\beta_{3 n}^{2}-\beta_{1 n}^{2}\right)+\left(e^{i\left(\beta_{2 n}+\beta_{3 n}\right)}+e^{i\left(\beta_{1 n}+\beta_{4 n}\right)}\right) \\
& \times\left(\beta_{1 n}^{2}-\beta_{4 n}^{2}\right)\left(\beta_{2 n}^{2}-\beta_{3 n}^{2}\right)=0 .
\end{aligned}
$$

For a fixed velocity, the linear natural frequencies of the travelling beam vary with flexural stiffness $\left(v_{f}\right)$. For specific values of flexural stiffness, the lower natural frequencies can be commensurable for a range of mean travelling velocity of the beam. These phenomena result in internal resonance in 


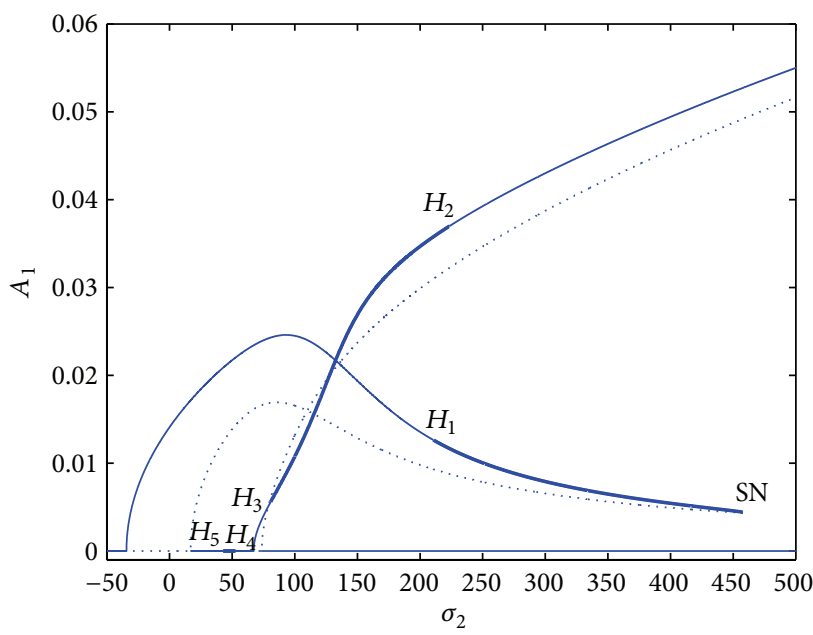

(a)

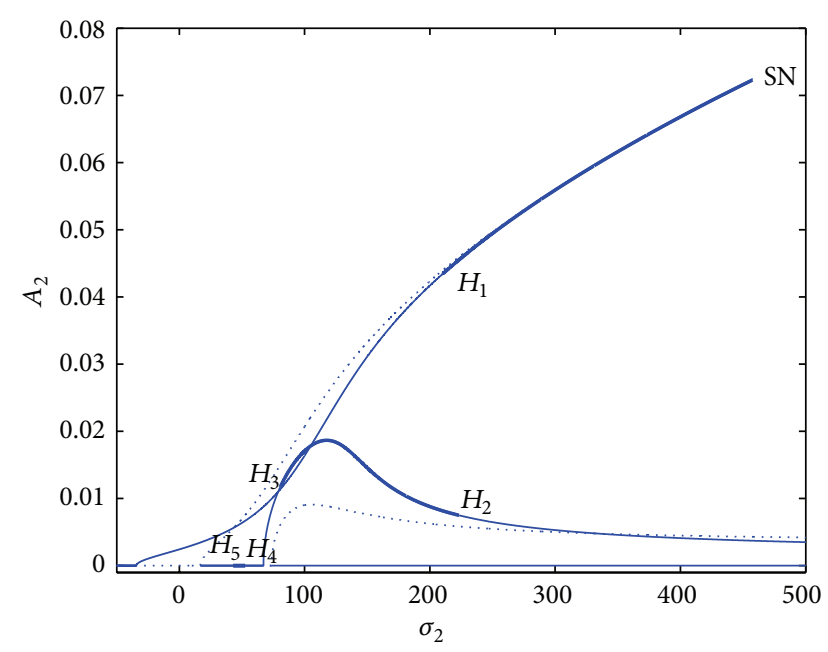

(b)

FIGURE 5: Frequency response curves for the (a) first mode and (b) second mode when the first mode is parametrically excited for the system parameters $\mu=0.1, \alpha=0, v_{1}=15, v_{l}=40$, and $\sigma_{1}=92.39$.

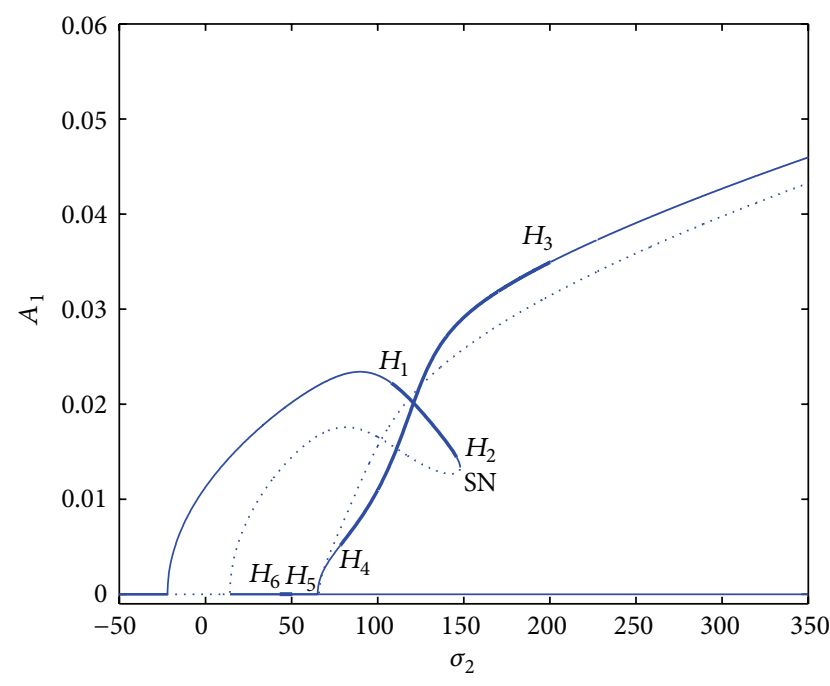

(a)

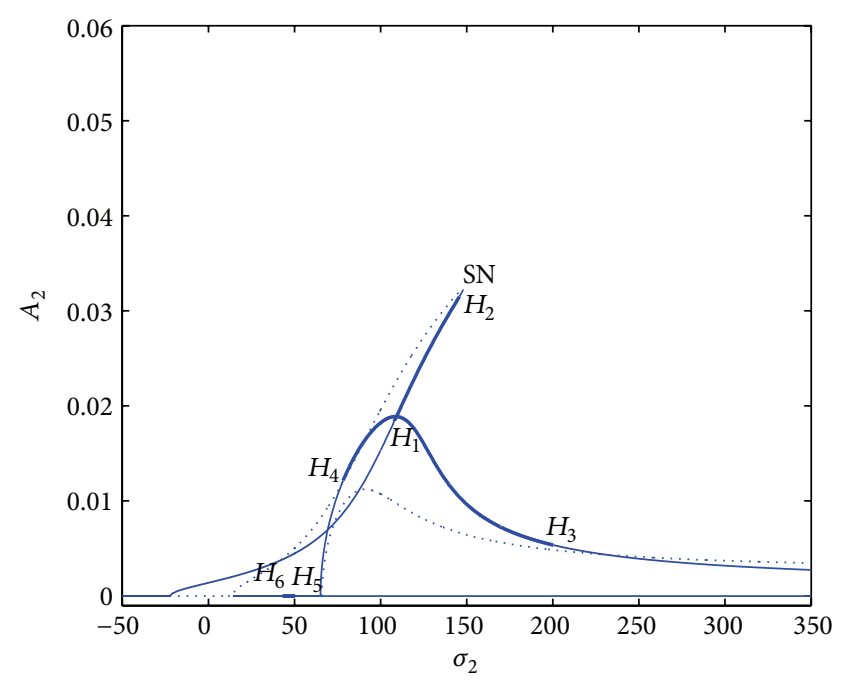

(b)

FIGURE 6: Frequency response curves for the (a) first mode and (b) second mode when the first mode is parametrically excited for the system parameters $\alpha=0.001, \mu=0, v_{1}=10, v_{l}=40$, and $\sigma_{1}=92.39$.

the system and nonlinear interaction between the involved lower modes. In the present investigation, a three-to-one internal resonance $\left(\omega_{2} \approx 3 \omega_{1}\right)$ is considered for a range of mean velocity of the beam. Also it is assumed that there is no other commensurable frequency relationship with higher modes. The case of principal parametric resonance of the first mode $\left(\Omega \approx 2 \omega_{1}\right)$ for subcritical flow velocities in presence of $3: 1$ internal resonance is analyzed in the present investigation. These first two modes are not in internal resonance with any higher modes, so the higher modes except the first two will decay with time due to the presence of damping and Coriolis terms present in the equation. Hence, the first two modes will contribute to the long term system response $[10,11]$. Consequently, we replace (11) with

$$
\begin{aligned}
w_{0}\left(T_{0}, T_{1}, x\right)= & A_{1}\left(T_{1}\right) \phi_{1}(x) e^{i \omega_{1} T_{0}} \\
& +A_{2}\left(T_{1}\right) \phi_{2}(x) e^{i \omega_{2} T_{0}}+c c .
\end{aligned}
$$

Now, we write the frequency relations for the internal resonance and principal parametric resonance as

$$
\omega_{2}=3 \omega_{1}+\varepsilon \sigma_{1}, \quad \Omega=2 \omega_{1}+\varepsilon \sigma_{2},
$$

where $\sigma_{1}$ and $\sigma_{2}$ are detuning parameters. It is worthy to note that $\Omega=\omega_{2}-\omega_{1}+\varepsilon\left(\sigma_{2}-\sigma_{1}\right)$, a combination 


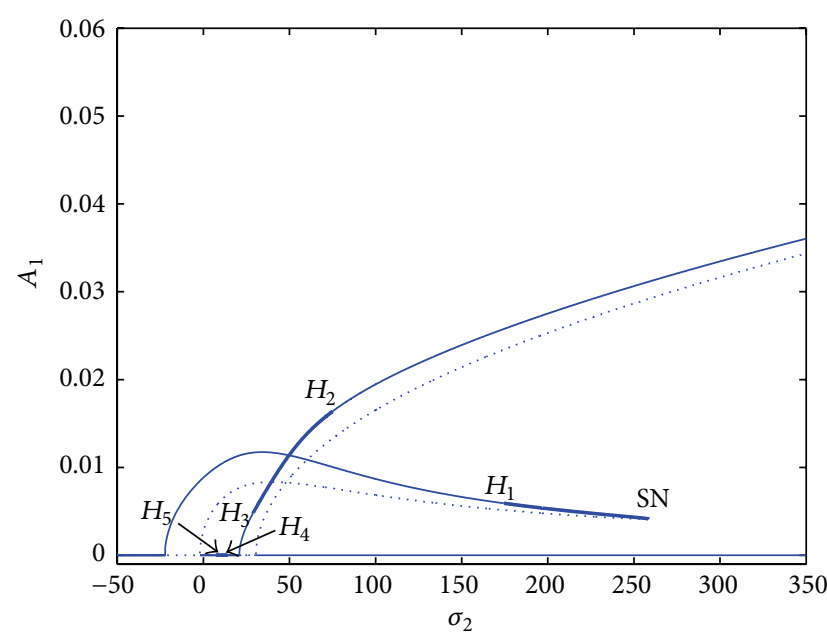

(a)

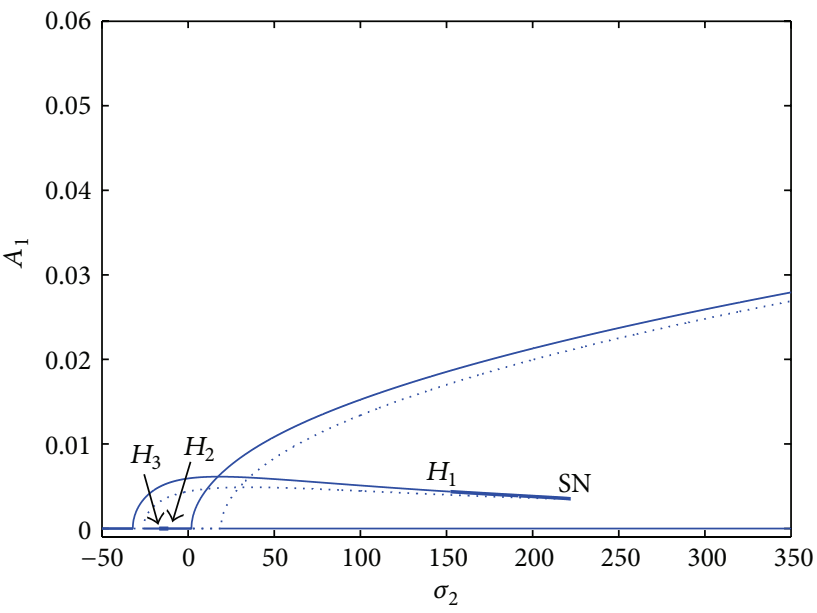

(c)

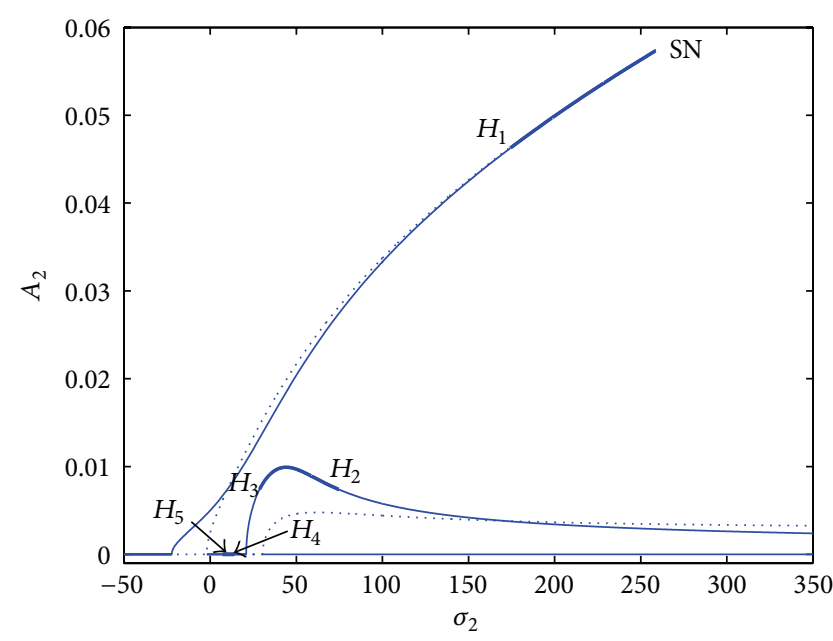

(b)

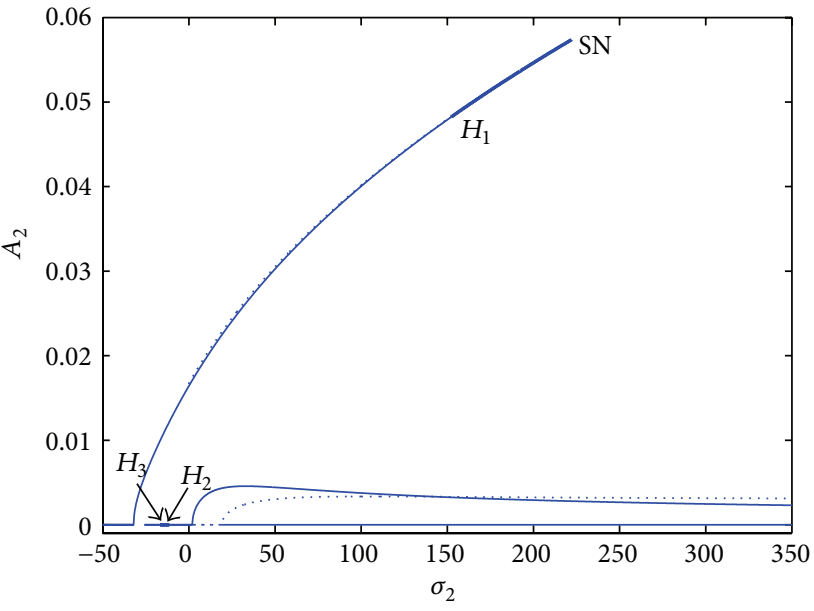

(d)

FIGURE 7: Effect of internal detuning parameter on the frequency response of (a) first mode and (b) second mode for system parameters $\mu=0.1, \alpha=0, v_{1}=10, v_{l}=40,(\mathrm{a}, \mathrm{b}) \sigma_{1}=20.320$, and $(\mathrm{c}, \mathrm{d}) \sigma_{1}=-27.680$.

parametric resonance of the difference type, is also activated simultaneously. Substituting (15) and (16) into (10), we get

$$
\begin{aligned}
D_{0}^{2} w_{1}+ & 2 v_{0} D_{0} w_{1}^{\prime}+\left(v_{0}^{2}-1\right) w_{1}^{\prime \prime}+v_{f}^{2} w_{1}^{\prime \prime \prime \prime} \\
= & \Gamma_{1} e^{i \omega_{1} T_{0}}+\Gamma_{2} e^{i\left(\omega_{1} T_{0}+\sigma_{1} T_{1}\right)}+\Gamma_{3} e^{i \omega_{1} T_{0}} \\
& +\Gamma_{4} e^{i\left(\omega_{1} T_{0}+\sigma_{1} T_{1}-\sigma_{2} T_{1}\right)}+\Gamma_{5} e^{i \omega_{2} T_{0}} \\
& +\Gamma_{6} e^{i\left(\omega_{2} T_{0}-\sigma_{1} T_{1}\right)}+\Gamma_{7} e^{i\left(\omega_{2} T_{0}+\sigma_{2} T_{1}-\sigma_{1} T_{1}\right)}+c c+\mathrm{NST},
\end{aligned}
$$

where the terms $\Gamma_{n}$ are defined in the Appendix section. NST stands for terms that do not produce secular or small divisor terms. As the homogeneous part of (17) with its associated boundary conditions has a nontrivial solution, the corresponding nonhomogeneous problem has a solution only if a solvability condition is satisfied [36,37]. This requires the right-hand side of (17) to be orthogonal to every solution of the adjoint homogeneous problem, which leads to the complex variable modulation equations for amplitude and phase:

$$
\begin{aligned}
2 A_{1}^{\prime}+ & 8 S_{1} A_{1}^{2} \bar{A}_{1}+8 S_{2} A_{1} A_{2} \bar{A}_{2}+8 g_{1} \bar{A}_{1}^{2} A_{2} e^{i \sigma_{1} T_{1}} \\
& +2 \mu C_{1} A_{1}+2 \alpha e_{1} A_{1}+2 K_{1} \bar{A}_{1} e^{i \sigma_{2} T_{1}} \\
& +2 K_{2} A_{2} e^{i\left(\sigma_{1}-\sigma_{2}\right) T_{1}}=0, \\
2 A_{2}^{\prime}+ & 8 S_{4} A_{2}^{2} \bar{A}_{2}+8 S_{3} A_{1} A_{2} \bar{A}_{1}+8 g_{2} A_{1}^{3} e^{-i \sigma_{1} T_{1}} \\
& +2 \mu C_{2} A_{2}+2 \alpha e_{2} A_{2}+2 K_{3} A_{1} e^{i\left(\sigma_{2}-\sigma_{1}\right) T_{1}}=0,
\end{aligned}
$$

where the prime denotes the differentiation with respect to slow time $T_{1}$ and $S_{i}, g_{i}, K_{i}, C_{i}$, and $e_{i}$ are defined in the Appendix section. Overbar indicates complex conjugate. The terms in the previous equations involving the internal frequency detuning parameter $\sigma_{1}$ are the contributions of the internal resonance in the system. 


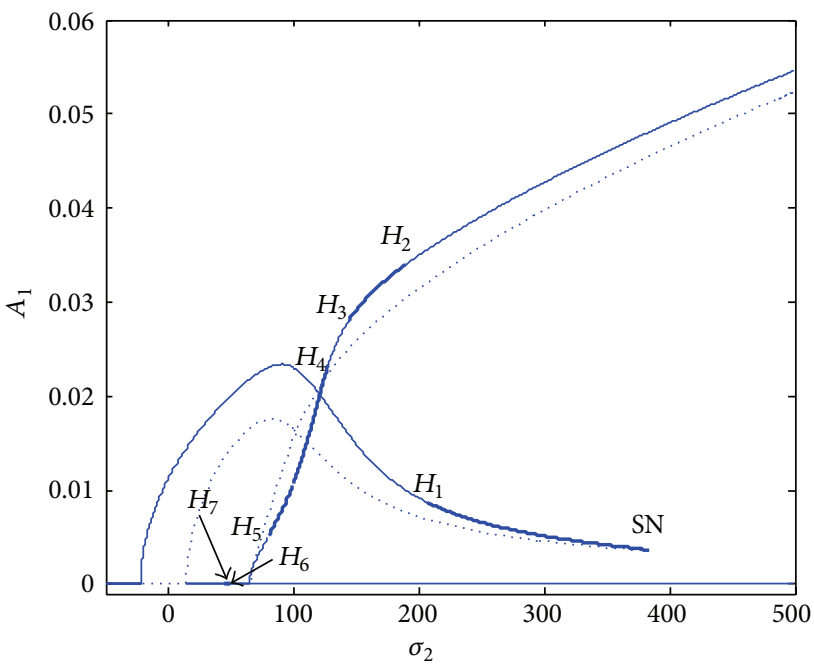

(a)

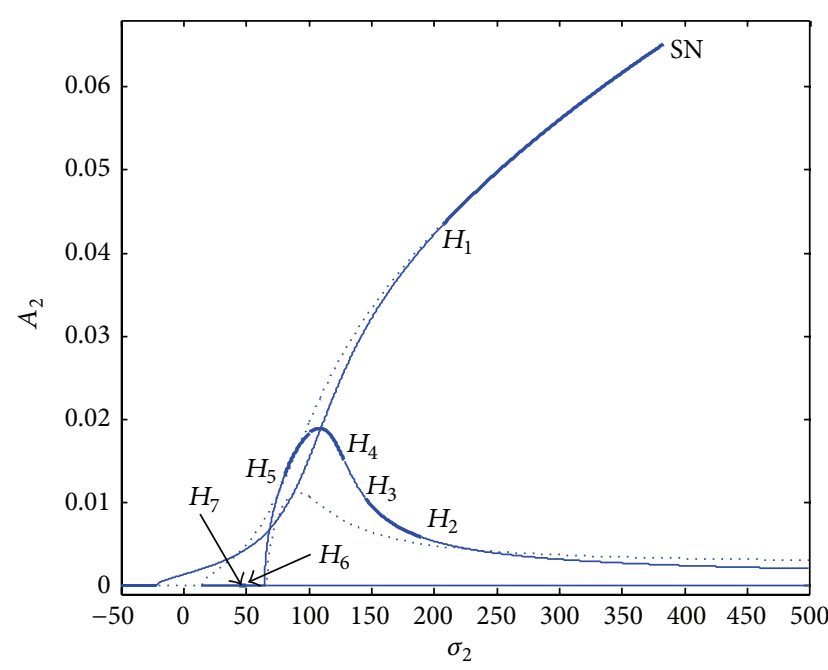

(b)

FiguRE 8: Effect of external damping parameter on the frequency response of first mode (a) and second mode (b) for $\mu=0.05, \alpha=0, v_{1}=10$, $v_{l}=40$, and $\sigma_{1}=92.39$.

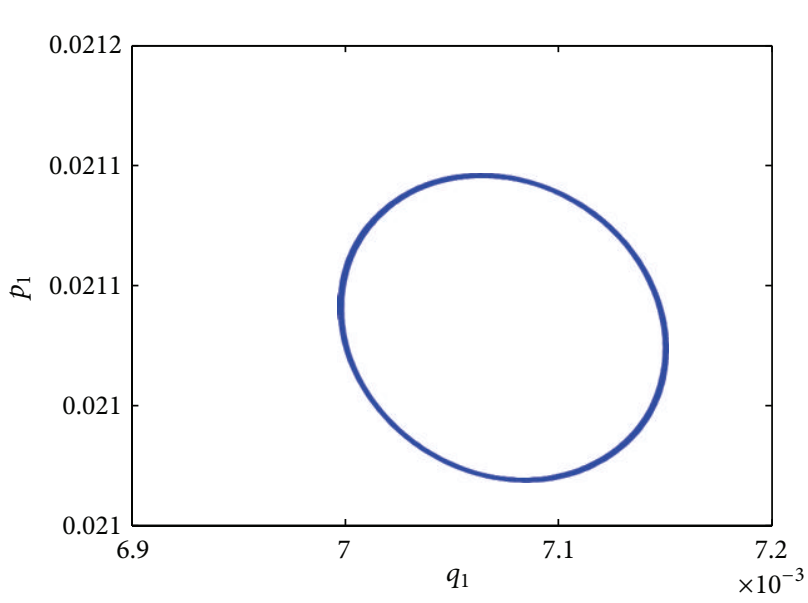

(a)

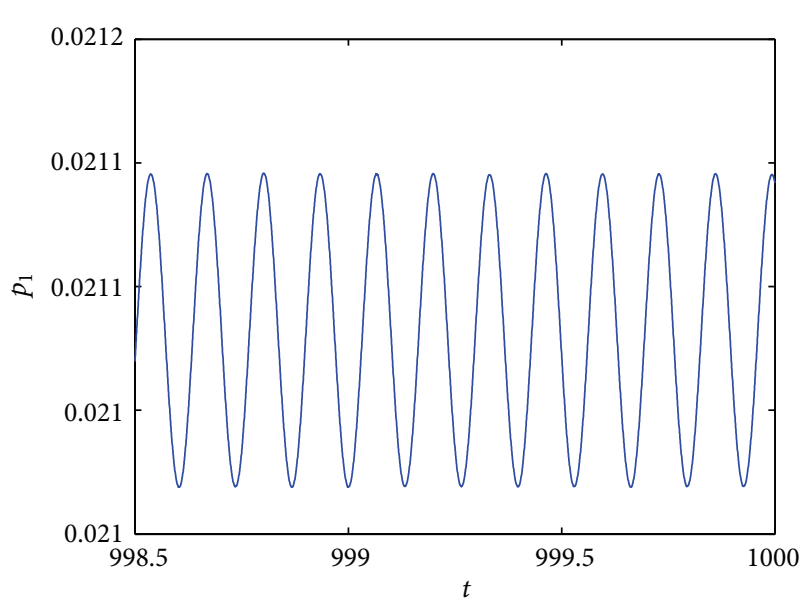

(c)

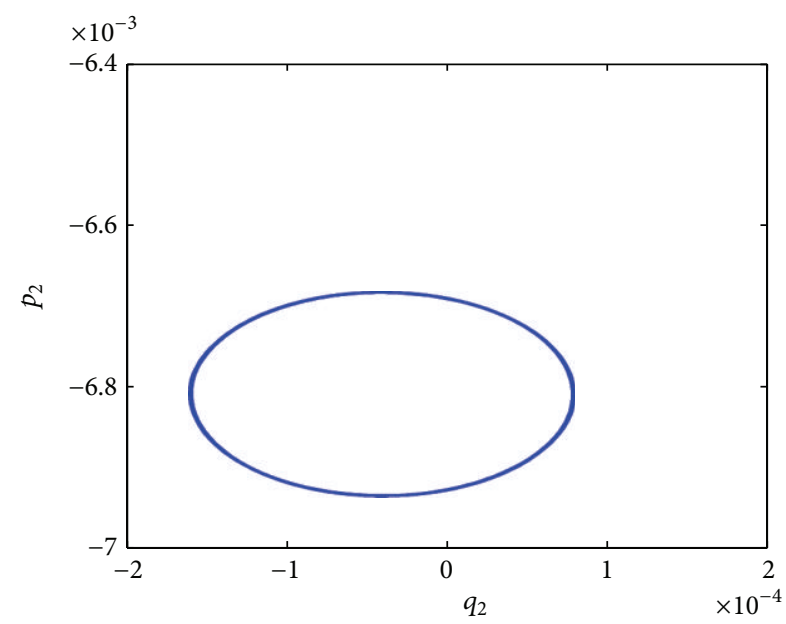

(b)

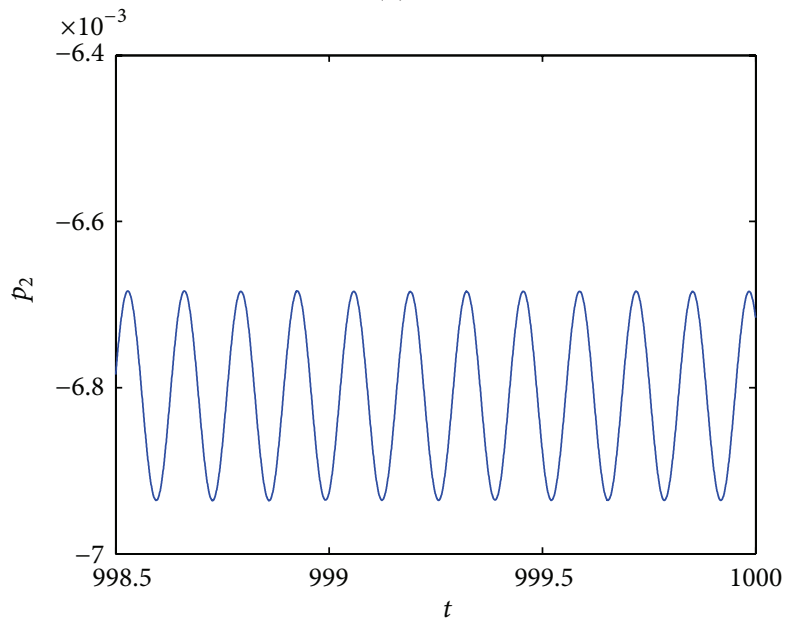

(d)

Figure 9: Phase portraits $(\mathrm{a}, \mathrm{b})$ and time histories $(\mathrm{c}, \mathrm{d})$ in the upper nontrivial stable branch of the frequency response curve of Figure 8 for $\sigma_{2}=68.2799, \mu=0.05, v_{1}=10, \alpha=0$, and $\sigma_{1}=92.39$. 


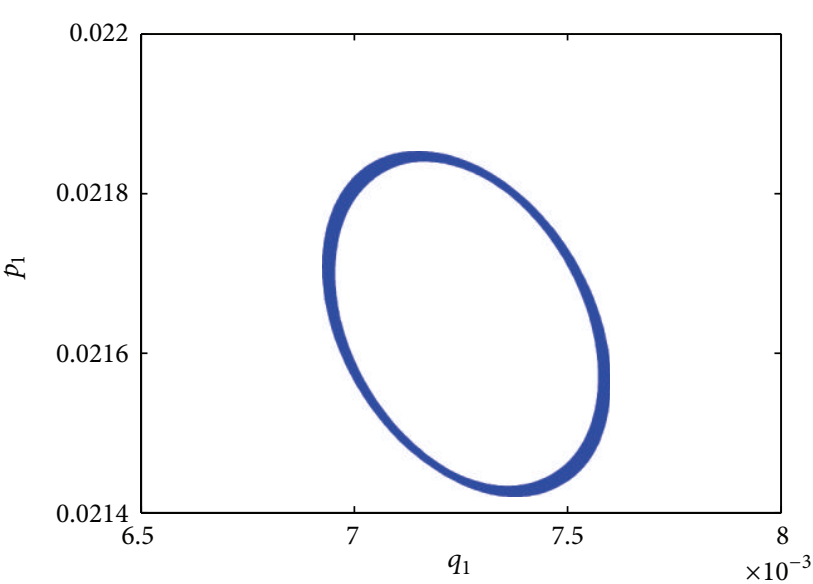

(a)

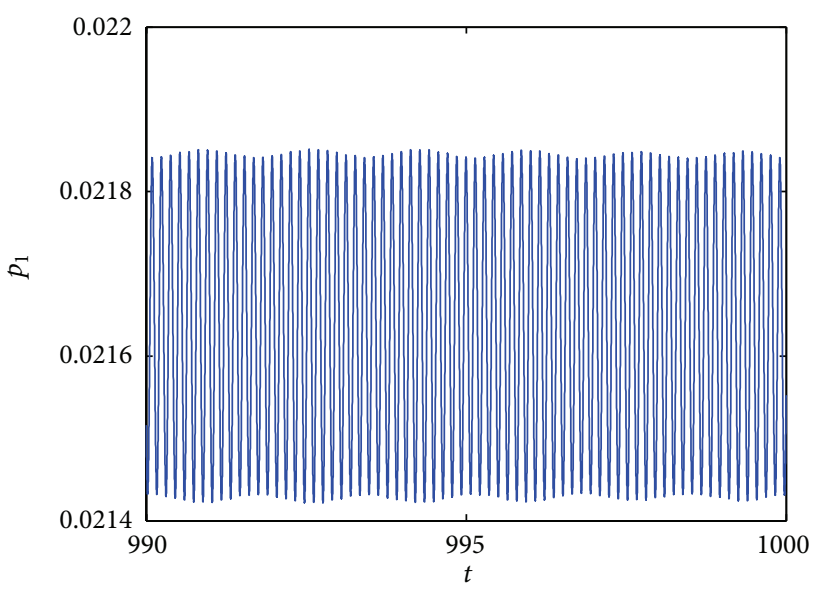

(c)

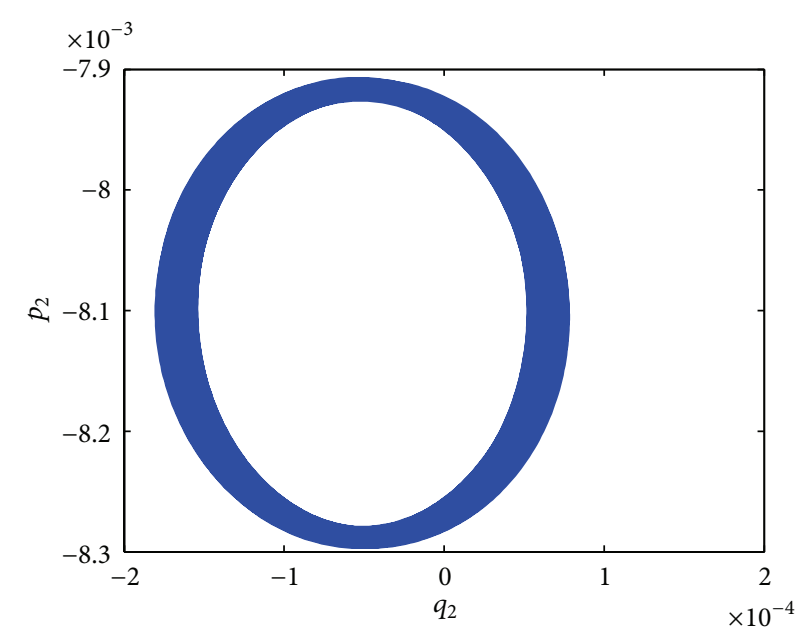

(b)

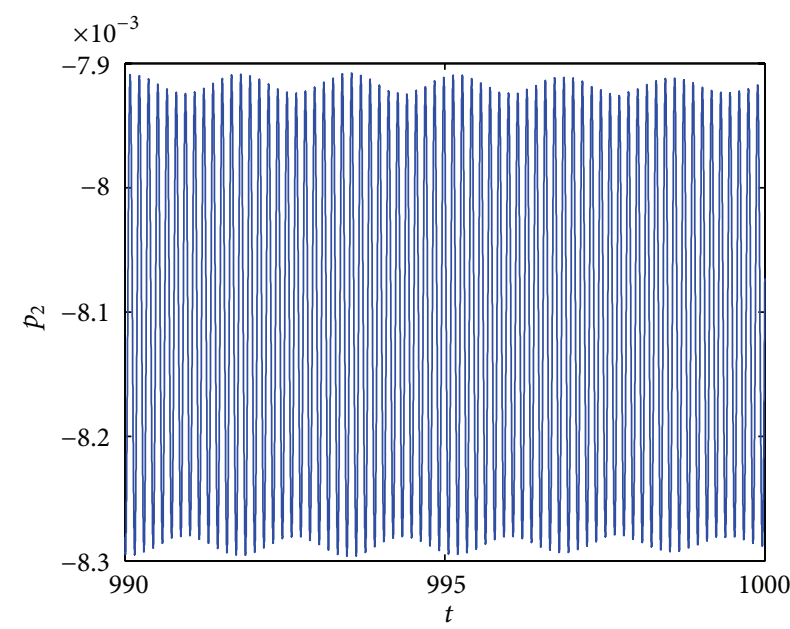

(d)

FIGURE 10: Phase portraits (a, b) and time histories (c, d) for $\sigma_{2}=75.2799, \mu=0.05, v_{1}=10, \alpha=0$, and $\sigma_{1}=92.39$.

\section{Stability and Bifurcations}

The evolutions of the equilibrium solutions and their stability and bifurcation analysis for principal parametric resonance of first mode are carried out from the modulation equation (18). The Cartesian transformation is used for the complex amplitude as

$$
A_{n}=\frac{1}{2}\left[p_{n}\left(T_{1}\right)-i q_{n}\left(T_{1}\right)\right] e^{i \lambda_{n}\left(T_{1}\right)}, \quad n=1,2 .
$$

Putting this in (18), simplifying by trigonometric manipulations, and separating the real and imaginary parts, we get the normalized reduced equations or the Cartesian form of modulation equations:

$$
\begin{aligned}
p_{1}^{\prime}= & -\vartheta_{1} q_{1}-S_{1 R}\left(p_{1}^{3}+p_{1} q_{1}^{2}\right)-S_{1 I}\left(p_{1}^{2} q_{1}+q_{1}^{3}\right) \\
& -S_{2 R}\left(p_{1} p_{2}^{2}+p_{1} q_{2}^{2}\right)-S_{2 I}\left(q_{1} p_{2}^{2}+q_{1} q_{2}^{2}\right) \\
& -g_{1 R}\left(p_{1}^{2} p_{2}-p_{2} q_{1}^{2}+2 p_{1} q_{1} q_{2}\right)
\end{aligned}
$$

$$
\begin{aligned}
& +g_{1 I}\left(2 p_{1} q_{1} p_{2}-p_{1}^{2} q_{2}+q_{1}^{2} q_{2}\right)-\mu C_{1 R} p_{1} \\
& -\mu C_{1 I} q_{1}-\alpha e_{1 R} p_{1}-\alpha e_{1 I} q_{1}-K_{1 R} p_{1} \\
& +K_{1 I} q_{1}-K_{2 R} p_{2}-K_{2 I} q_{2}, \\
q_{1}^{\prime}= & \vartheta_{1} p_{1}+S_{1 I}\left(p_{1}^{3}+p_{1} q_{1}^{2}\right)-S_{1 R}\left(p_{1}^{2} q_{1}+q_{1}^{3}\right) \\
& -S_{2 R}\left(q_{1} p_{2}^{2}+q_{1} q_{2}^{2}\right)+S_{2 I}\left(p_{1} p_{2}^{2}+p_{1} q_{2}^{2}\right) \\
& +g_{1 R}\left(2 p_{1} q_{1} p_{2}-p_{1}^{2} q_{2}+q_{1}^{2} q_{2}\right) \\
& +g_{1 I}\left(2 p_{1} q_{1} q_{2}+p_{1}^{2} p_{2}-p_{2} q_{1}^{2}\right)+K_{1 R} q_{1}+K_{1 I} p_{1} \\
& -K_{2 R} q_{2}+K_{2 I} p_{2}-\mu C_{1 R} q_{1}+\mu C_{1 I} p_{1} \\
& -\alpha e_{1 R} q_{1}+\alpha e_{1 I} p_{1}, \\
p_{2}^{\prime}= & -\vartheta_{2} q_{2}-S_{4 R}\left(p_{2}^{3}+p_{2} q_{2}^{2}\right)-S_{4 I}\left(q_{2}^{3}+p_{2}^{2} q_{2}\right) \\
& -S_{3 R}\left(p_{1}^{2} p_{2}+p_{2} q_{1}^{2}\right)-S_{3 I}\left(p_{1}^{2} q_{2}+q_{1}^{2} q_{2}\right)
\end{aligned}
$$




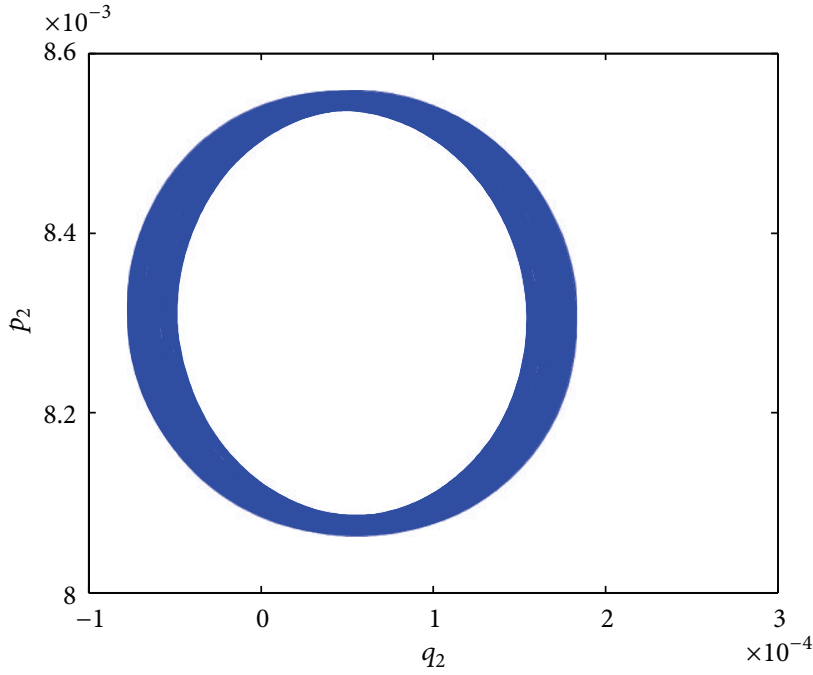

(a)

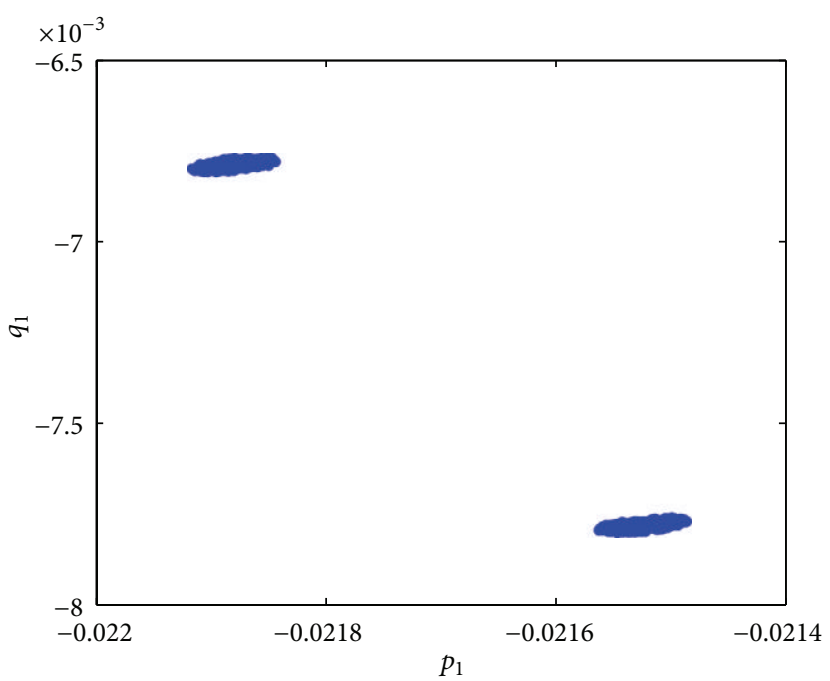

(c)

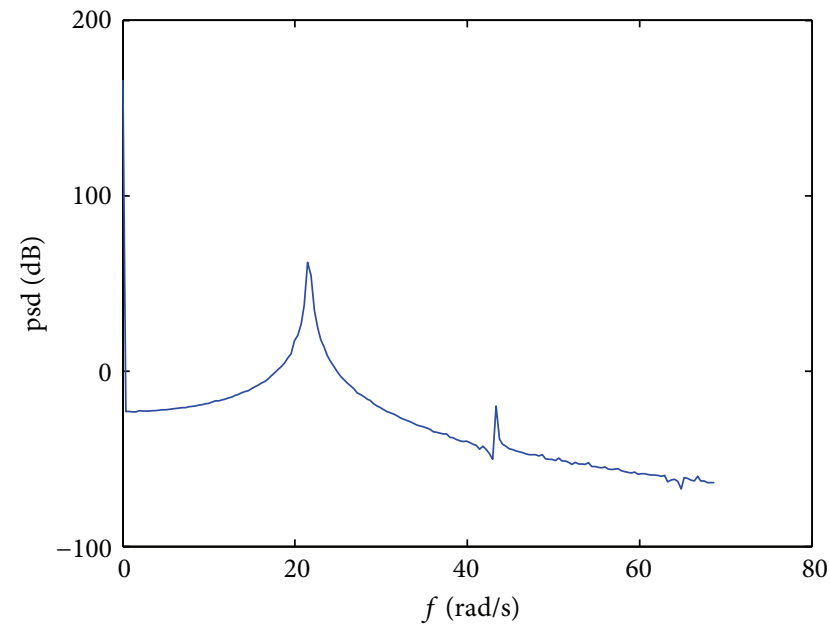

(b)

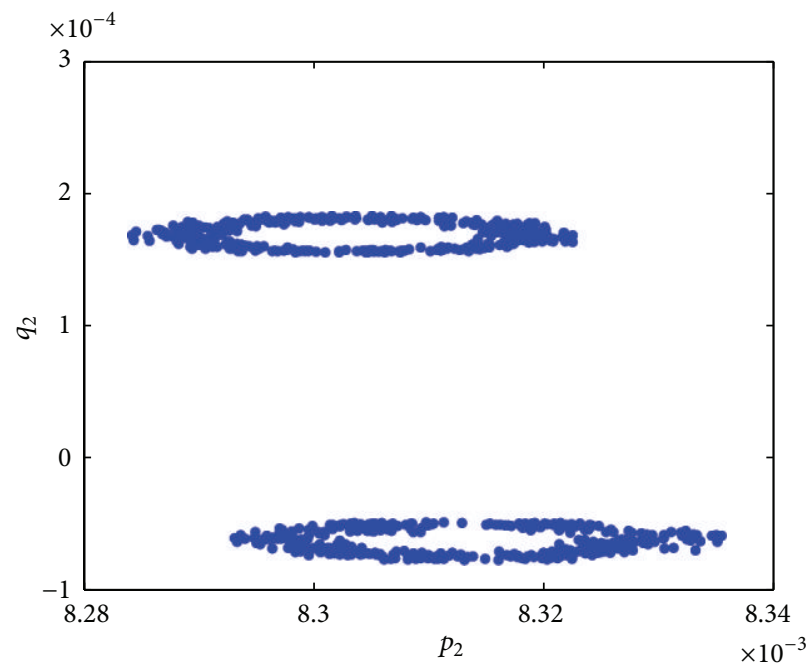

(d)

FIGURE 11: Phase portrait (a), FFT power spectra (b), and Poincare maps (c, d) for $\sigma_{2}=76.2799, \mu=0.05, v_{1}=10$, and $\alpha=0, \sigma_{1}=92.39$.

$$
\begin{aligned}
& -g_{2 R}\left(p_{1}^{3}-3 p_{1} q_{1}^{2}\right)+g_{2 I}\left(q_{1}^{3}-3 p_{1}^{2} q_{1}\right) \\
& -K_{3 R} p_{1}-K_{3 I} q_{1}-\mu C_{2 R} p_{2}-\mu C_{2 I} q_{2} \\
& -\alpha e_{2 R} p_{2}-\alpha e_{2 I} q_{2}, \\
q_{2}^{\prime}= & -\vartheta_{2} p_{2}-S_{4 R}\left(q_{2}^{3}+p_{2}^{2} q_{2}\right)+S_{4 I}\left(p_{2}^{3}+p_{2} q_{2}^{2}\right) \\
& -S_{3 R}\left(p_{1}^{2} q_{2}+q_{1}^{2} q_{2}\right)+S_{3 I}\left(p_{1}^{2} p_{2}+p_{2} q_{1}^{2}\right) \\
& +g_{2 R}\left(q_{1}^{3}-3 p_{1}^{2} q_{1}\right)+g_{2 I}\left(p_{1}^{3}-3 p_{1} q_{1}^{2}\right) \\
& -K_{3 R} q_{1}+K_{3 I} p_{1}-\mu C_{2 R} q_{2}+\mu C_{2 I} p_{2} \\
& -\alpha e_{2 R} q_{2}+\alpha e_{2 I} p_{2},
\end{aligned}
$$

where

$$
\vartheta_{1}=0.5 \sigma_{2}, \quad \vartheta_{2}=1.5 \sigma_{2}-\sigma_{1} .
$$

The previous equations are perturbed to evaluate the stability. The perturbed equation is

$$
\left\{\Delta p_{1}^{\prime} \Delta q_{1}^{\prime} \Delta p_{2}^{\prime} \Delta q_{2}^{\prime}\right\}^{T}=\left[J_{c}\right]\left\{\Delta p_{1} \Delta q_{1} \Delta p_{2} \Delta q_{2}\right\}^{T},
$$

where $T$ denotes transpose and $\left[J_{c}\right]$ is the Jacobian matrix whose eigenvalues determine the stability and bifurcation of the system. The stability boundary for trivial state is obtained by setting $p_{1}=q_{1}=p_{2}=q_{2}=0$. The nonlinear steady state response behavior of the system is obtained from the normalized reduced equation (20) by setting $p_{1}^{\prime}=q_{1}^{\prime}=$ $p_{2}^{\prime}=q_{2}^{\prime}=0$ and then solving the resulting set of nonlinear 


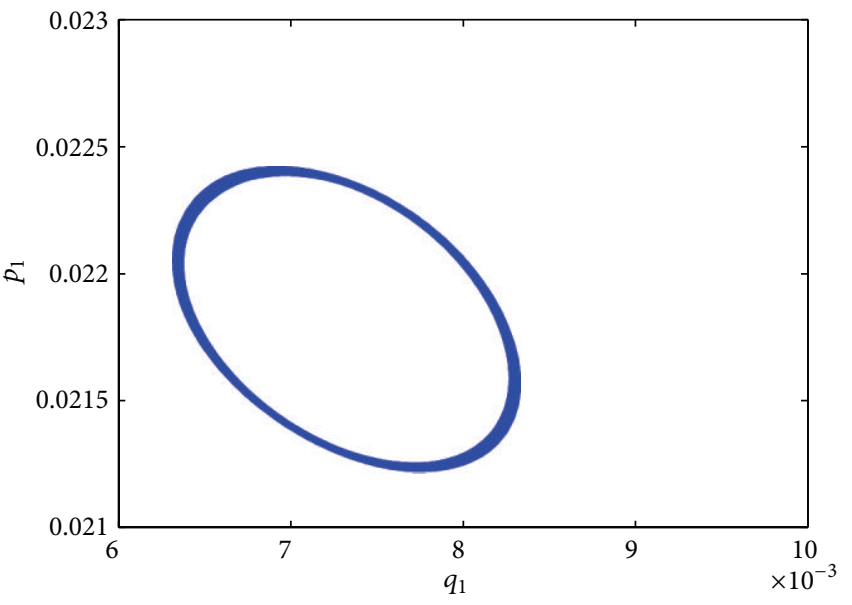

(a)

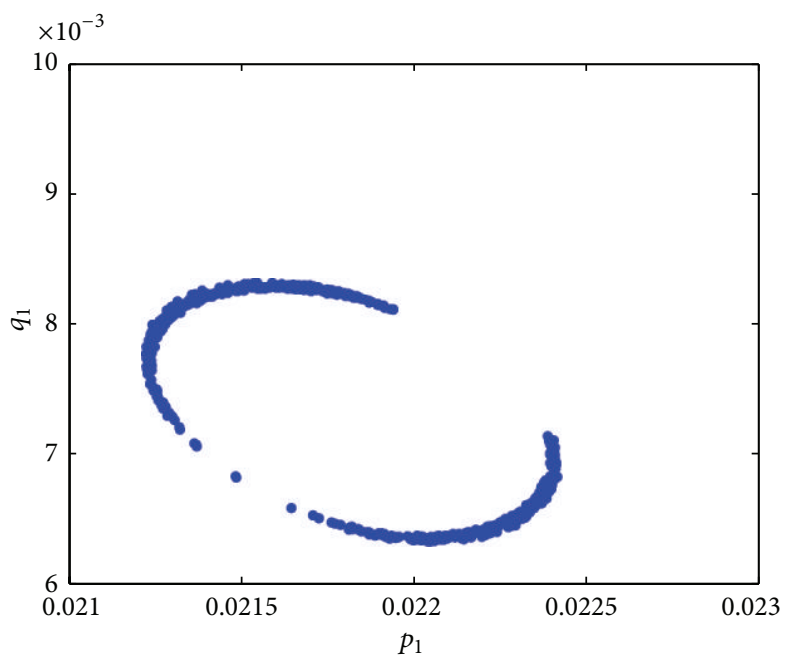

(c)

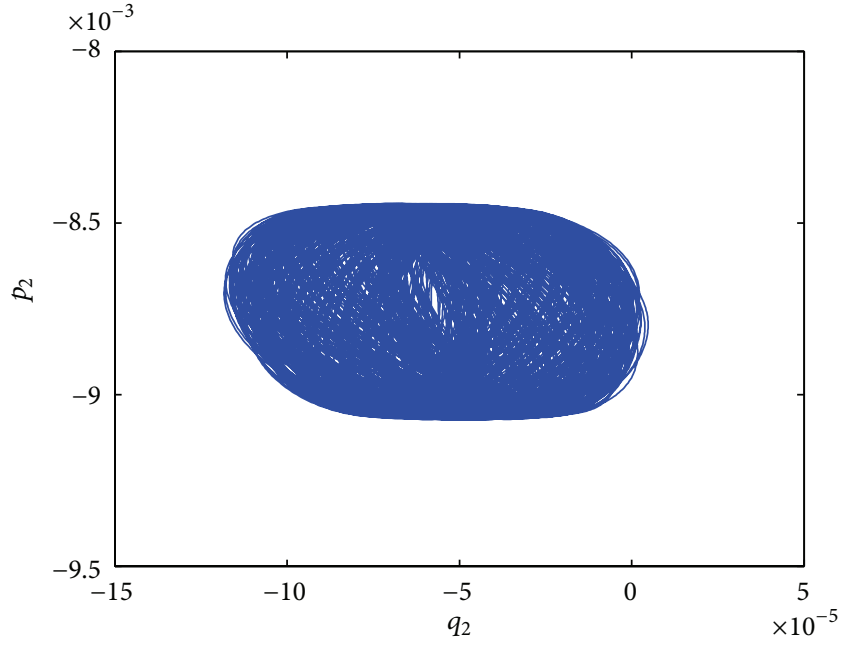

(b)

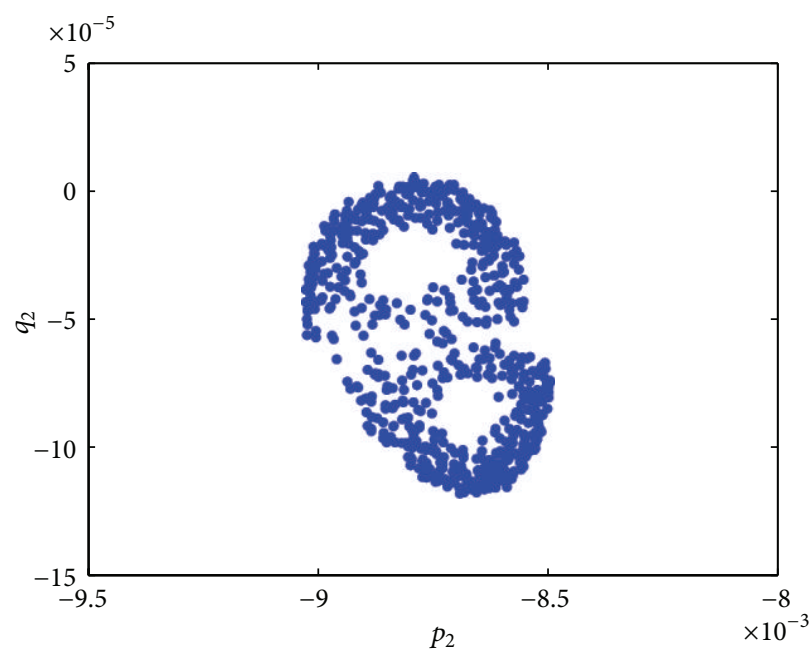

(d)

Figure 12: Phase portraits (a, b) and Poincare maps (c, d) for $\sigma_{2}=78.2799, \mu=0.05, v_{1}=10, \alpha=0$, and $\sigma_{1}=92.39$.

algebraic equations. The same set of equations is also used for the analysis of stability and bifurcation of trivial as well as nontrivial solutions. The analysis for dynamic solutions is carried out by numerically integrating (20) with different combinations of system parameters.

\section{Results and Discussions}

The natural frequencies of the beam are numerically evaluated at different mean velocities $\left(v_{0}\right)$ with flexural stiffness $v_{f}=0.2$ by simultaneous solution of dispersive relation (13) and support condition (14). The results are presented in Table 1. It is noticed that, at nondimensional mean velocity $v_{0}=0.513$, the natural frequency of second mode is approximately equal to three times that of the first mode implying the existence of $3: 1$ internal resonance. It is also noticed that there are no other commensurable frequency relationships involving higher modes. Therefore, nonlinear interaction among higher modes is ruled out. The investigation is limited to the case of principal parametric resonance of first mode, that is, $\Omega \approx 2 \omega_{1}$, in presence of internal resonance in the subcritical mean velocity regime of a travelling beam.

The trivial state stability boundary shown in Figure 2 is plotted in terms of principal parametric frequency detuning $\left(\sigma_{2}\right)$ and amplitude of fluctuating velocity component $\left(v_{1}\right)$ for system parameters $v_{f}=0.2, v_{l}=40, v_{0}=0.7$, $\omega_{1}=2.7388$, and $\omega_{2}=9.1403$ and for different damping values. The book keeping parameter is taken as $\varepsilon=0.01$, and the corresponding internal frequency detuning parameter is assumed to be $\sigma_{1}=92.39$. The region inside the boundary denotes instability. Higher values of damping have the effect of raising and narrowing the instability zones. It is revealed that the effect of material damping $(\alpha)$ raises and narrows the instability zone more compared to that of viscous damping $(\mu)$ on the trivial state stability boundary. However, this trivial state stability plot may not reveal completely the real system behavior, because in the unstable zone of the trivial stability plot, the system may have stable nontrivial 


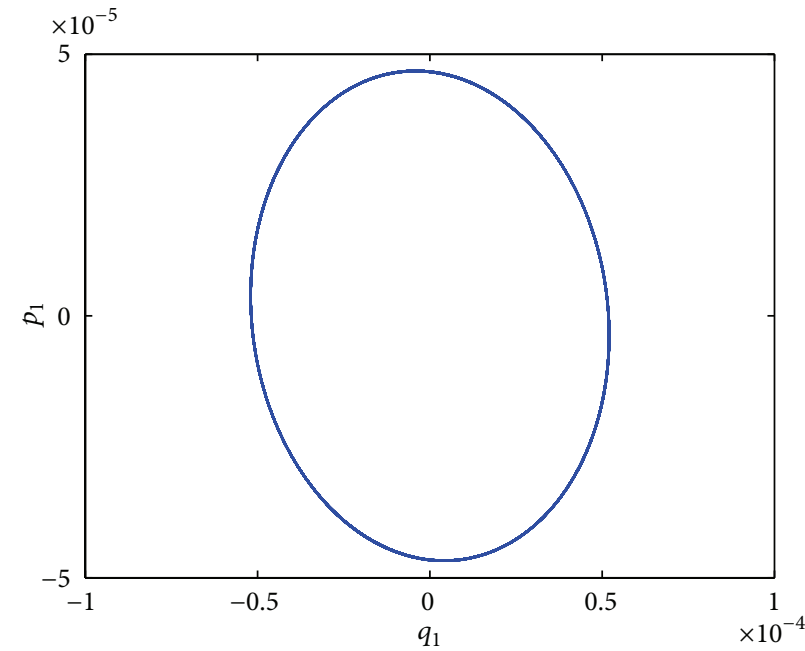

(a)

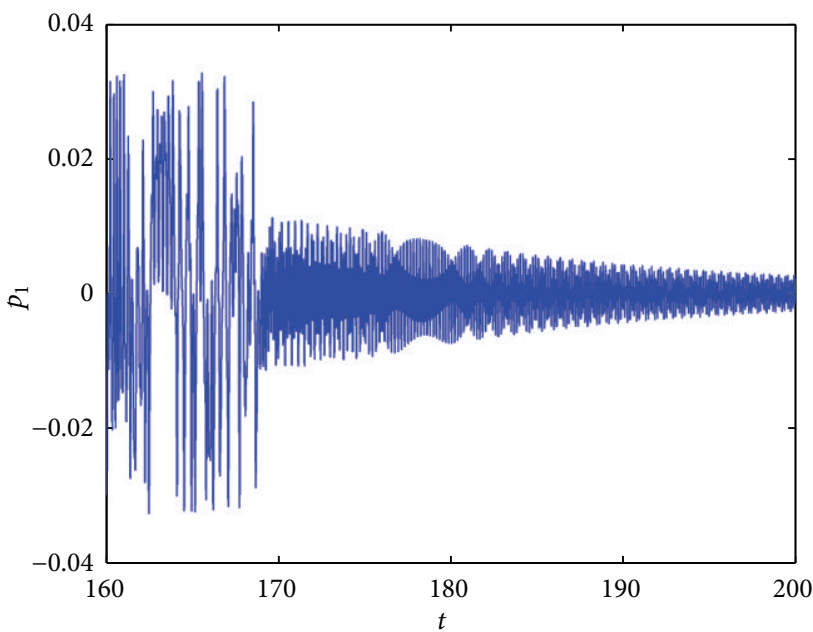

(c)

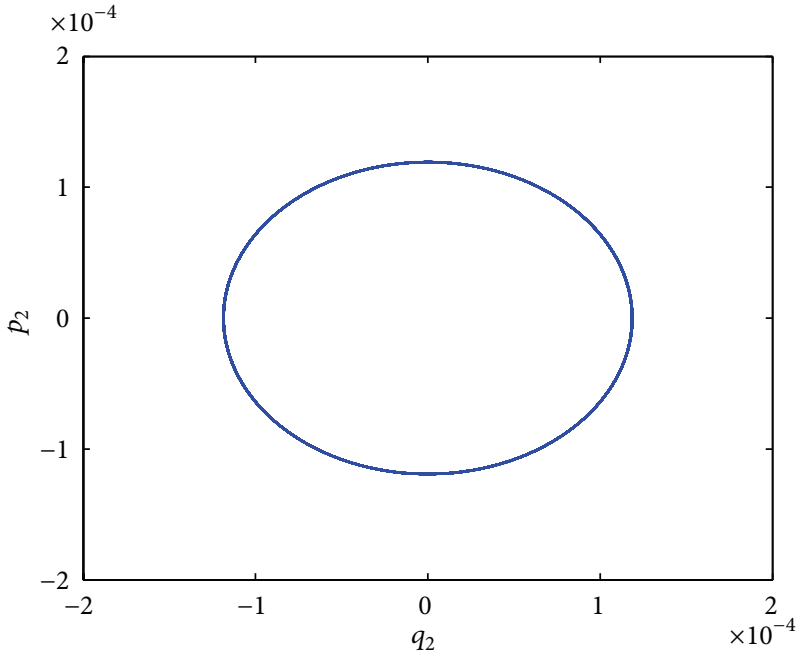

(b)

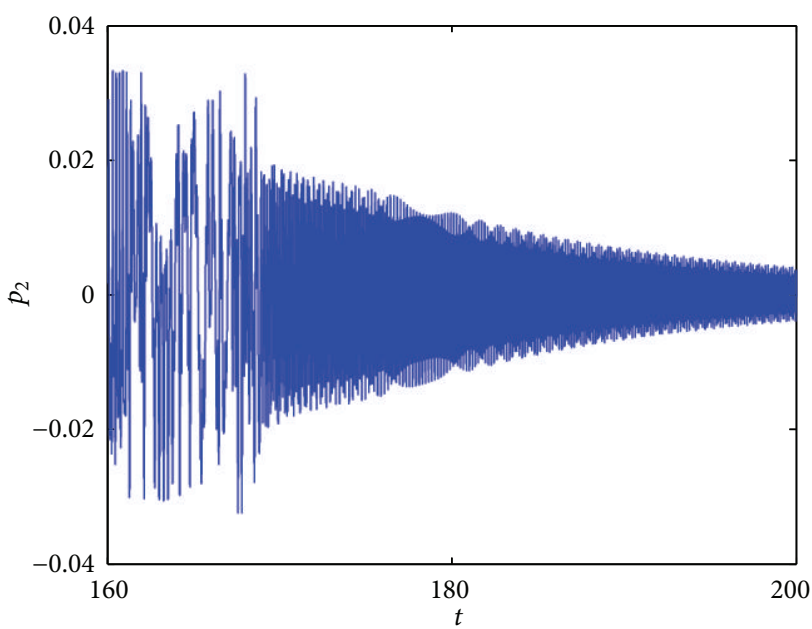

(d)

FIGURE 13: Phase portraits (a, b) and time histories (c, d) for $\sigma_{2}=115.7201, \mu=0.05, v_{1}=10, \alpha=0$, and $\sigma_{1}=92.39$.

equilibrium solution or stable dynamic solution like periodic or quasiperiodic solution. In addition, there is a possibility of chaotic solutions or multiple stable solutions. For this reason, it is required to carry out the dynamic analysis as well as the stability and bifurcation study of equilibrium solutions of the system.

5.1. Stability and Bifurcations of Equilibrium Solutions. Continuation algorithm is used to determine the nonlinear steady state response by solving the set of algebraic equations generated after setting $p_{i}^{\prime}=q_{i}^{\prime}=0$ in the normalized reduced equation (20). The stability and bifurcation of the equilibrium solutions are obtained from the eigenvalues of the Jacobean matrix at each point of the solution. In order to validate the results obtained by the present analysis, the frequency response and amplitude response curves of Chin et al. [16] are generated once again using continuation algorithm. They are shown in Figure 3, and the results are found to be in good agreement. Since the frequency and amplitude response curves are symmetrical about $\sigma_{2}$ and $v_{1}$ axes, respectively, only positive sides of the response curves are shown.

Frequency response curves are obtained against variation in frequency detuning parameter $\sigma_{2}$ for first and second mode for $\mu=0.1, \alpha=0, v_{1}=10, v_{l}=40$, and $\sigma_{1}=92.39$ and are shown in Figure 4. The normal continuous lines in the figure represent stable equilibrium solutions, the bold lines represent unstable foci, and the dotted lines denote saddles. Different parameter values for characteristic points on different branches of the plot are indicated in Table 2. The response curves exhibit a hardening-spring type of nonlinearity. With increase in $\sigma_{2}$ from a small value, the trivial stable solution loses stability at $\sigma_{2}=-22.071$, through supercritical pitchfork bifurcation, and results in a two-mode nontrivial stable equilibrium solution. It is observed that the amplitude of the first mode increases initially then decreases, but the amplitude of second mode increases monotonically. When the value of $\sigma_{2}$ increases, the equilibrium solution becomes unstable at $H_{1}\left(\sigma_{2}=206.588\right)$ through Hopf bifurcation, and 


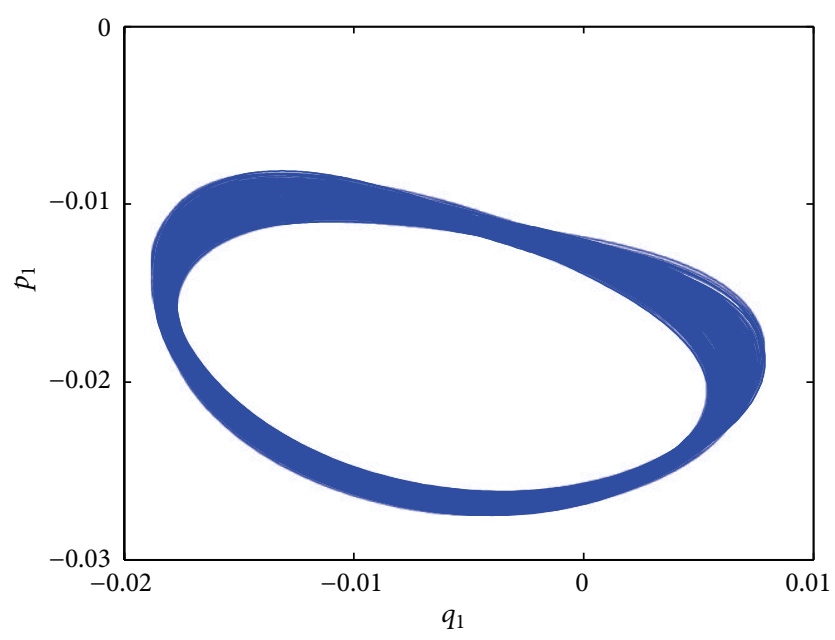

(a)

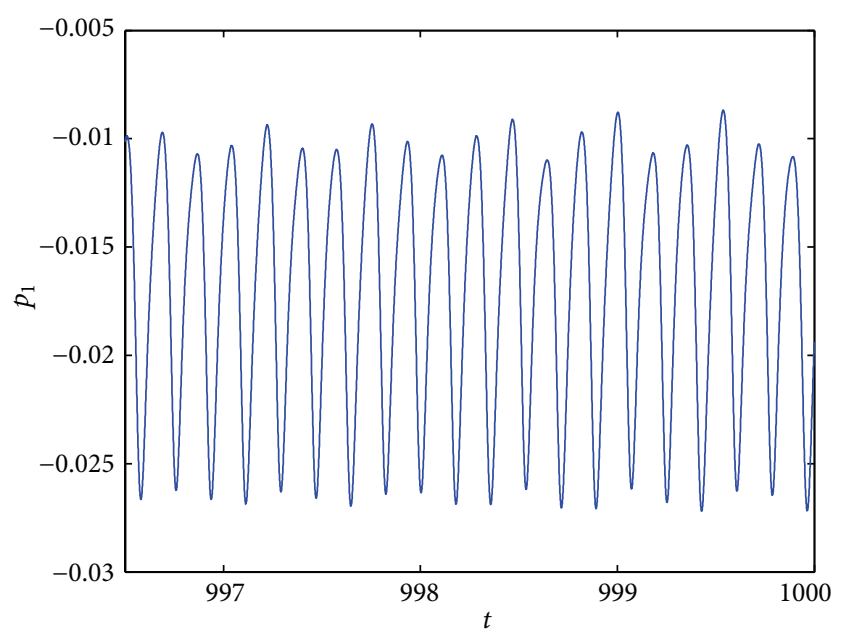

(c)

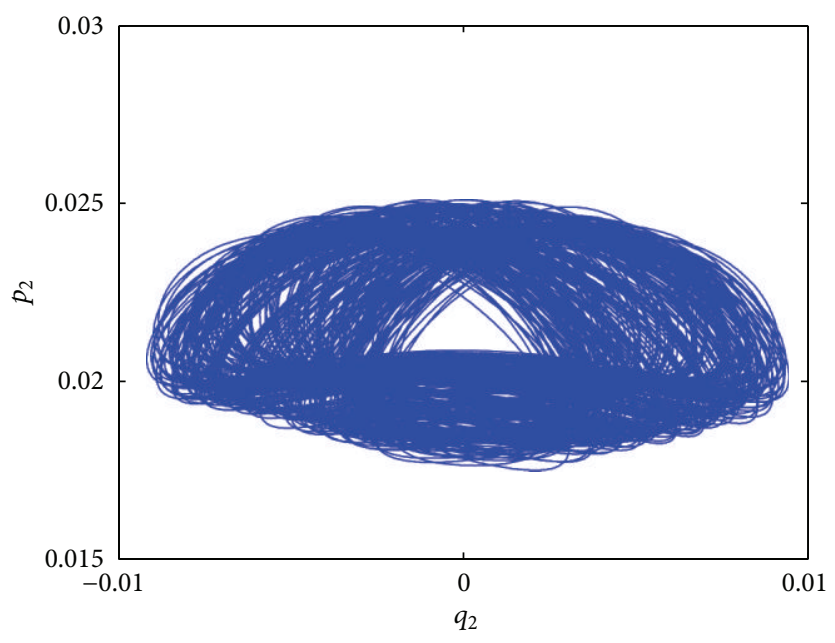

(b)

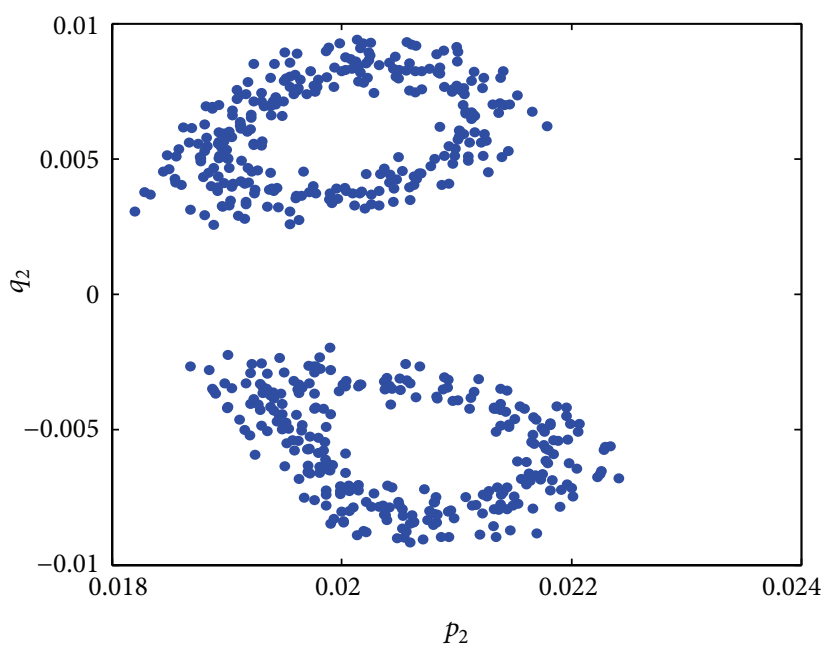

(d)

Figure 14: Phase portraits (a, b), time trace (c), and Poincare map (d) for $\sigma_{2}=116.4201, \mu=0.05, v_{1}=10$, and $\alpha=0, \sigma_{1}=92.39$.

out of two pairs of complex conjugate eigenvalues, one pair crosses the imaginary axis from the left half of the complex plane to the right half. With further increase of $\sigma_{2}$, the same state continues until a saddle node bifurcation occurs at $\mathrm{SN}\left(\sigma_{2}=301.873\right)$, where the system response jumps to one of the two stable equilibrium branches, one trivial and the other nontrivial, depending on the initial conditions as the solution converges to the closer equilibrium state as per the concept of region of attraction. With further increase in frequency detuning parameter, amplitude of the first mode increases monotonically along the nontrivial branch, whereas the amplitude of second mode decreases continuously. Thus the amplitude of the indirectly excited second mode is limited to a fixed higher magnitude, and for high values of $\sigma_{2}$, it becomes stagnant at fixed low amplitude while there is no such limitation for the directly excited first mode.

When the detuning parameter decreases from a high value, the system follows either trivial or nontrivial stable equilibrium path, depending on the initial conditions. If the solution is nontrivial, with decrease of $\sigma_{2}$ value, the nontrivial stable branch loses stability via Hopf bifurcation at $H_{2}\left(\sigma_{2}=\right.$ 190.033) and regains stability via a reverse Hopf bifurcation at $H_{3}\left(\sigma_{2}=144.502\right)$. When the detuning parameter is further decreased, again the system loses stability via Hopf bifurcation at $H_{4}\left(\sigma_{2}=127.98\right)$ and regains stability via a reverse Hopf bifurcation at $H_{5}\left(\sigma_{2}=80.408\right)$ on the same path. With further decrease in frequency detuning parameter, the nontrivial stable equilibrium branch merges with stable trivial equilibrium solution, the system losing and regaining the stability at $H_{6}\left(\sigma_{2}=49.474\right)$ and $H_{7}\left(\sigma_{2}=44.608\right)$, respectively. At $\sigma_{2}=14.190$, the trivial equilibrium solution loses stability via subcritical/reverse pitchfork bifurcation and results in a jump of the response to the stable nontrivial branch of the solution. Again with further decrease of frequency detuning, the nontrivial stable solution branch loses stability through pitchfork bifurcation at $\sigma_{2}=-22.071$, giving the way to trivial solution. The directly excited first mode dominates the indirectly excited second mode. 


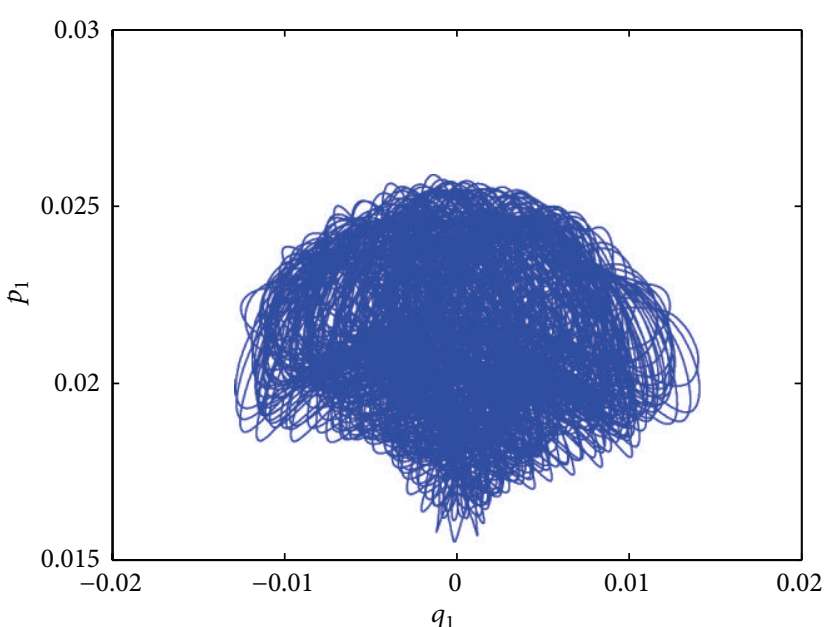

(a)

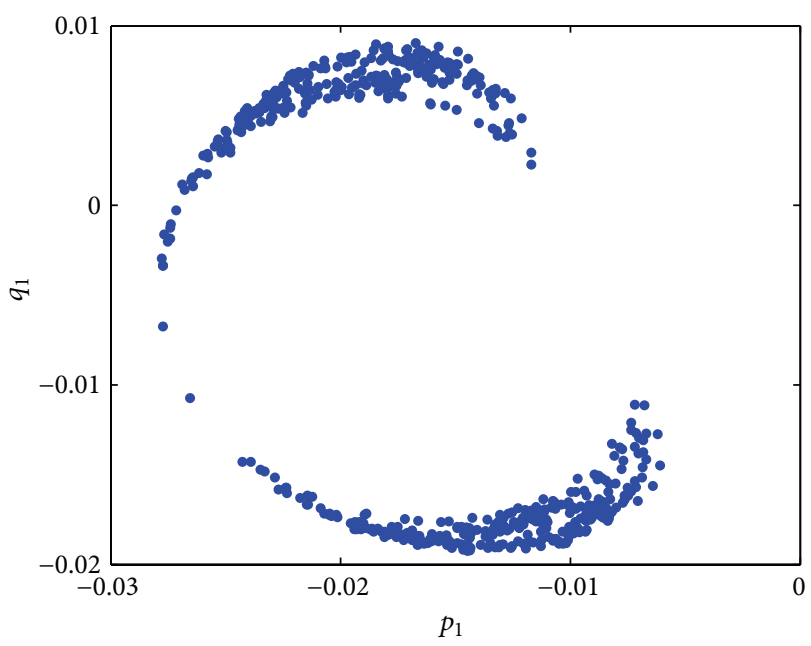

(c)

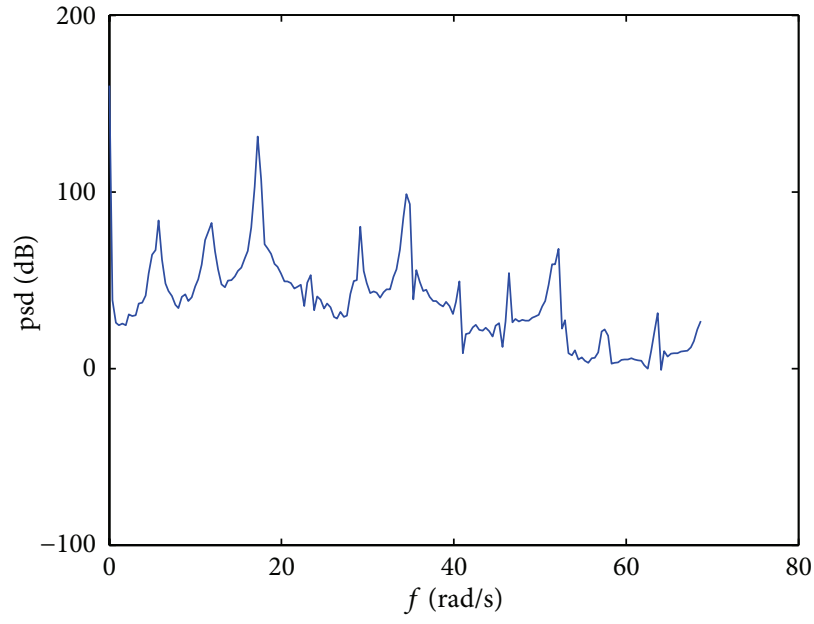

(b)

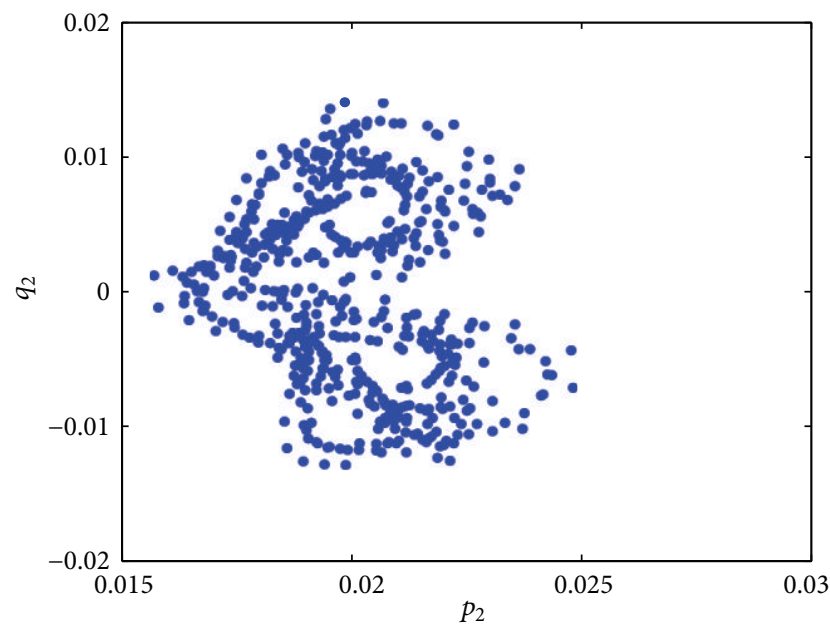

(d)

FIgURE 15: Phase portrait (a), FFT power spectra (b), and Poincare maps (c, d) for $\sigma_{2}=117.4201, \mu=0.05, v_{1}=10, \alpha=0$, and $\sigma_{1}=92.39$.

Figure 5 shows the frequency response curves for first and second mode of the system for higher amplitude of the fluctuating velocity component $\left(v_{1}\right)$. The system parameters considered are $\mu=0.1, \alpha=0, v_{1}=15, v_{l}=40$, and $\sigma_{1}=92.39$. Even though the solution curves are similar in shape to the curves obtained in case of lower amplitude of excitation $\left(v_{1}=10\right)$ as shown in Figure 4, the jump phenomena at the saddle node bifurcation ( $\mathrm{SN}$ ) occur at higher value of the detuning parameter $\left(\sigma_{2}=457.7476\right)$ compared to the previous case $\left(\sigma_{2}=301.8730\right.$ (Figure 4$)$ ). In addition, unstable zone in trivial solution gets broadened which is commensurate with the trivial state stability plot.

Figure 6 shows typical frequency response curves for two modes considering the effect of the internal damping for system parameters $\mu=0, \alpha=0.001, v_{1}=10, v_{l}=$ 40 , and $\sigma_{1}=92.39$. The strength of nonlinear interaction due to internal resonance gets weakened due to internal damping (Figure 6) compared to the case of external damping (Figure 4). The influence of internal detuning parameter $\left(\sigma_{1}\right)$ on the frequency response is shown in Figure 7. It is evident that the decrease in internal detuning parameter $\left(\sigma_{1}\right)$ to 20.320 (Figures $7(\mathrm{a})$ and $7(\mathrm{~b})$ ) and -27.680 (Figures 7(c) and $7(\mathrm{~d})$ ) from 92.390 (Figure 4) weakens the strength of nonlinear interaction due to three-to-one internal resonance. The amplitude of the directly excited first mode decreases more pronouncedly than the indirectly excited second mode. Beside this, the number of Hopf bifurcation points on the upper nontrivial curve decreases from four for $\sigma_{1}=92.39$ to two for $\sigma_{1}=20.320$ and totally vanishes for $\sigma_{1}=-27.680$, and also there is decreasing trend in $\sigma_{2}$ value at which saddle node bifurcation occurs. Figure 8 shows the effect of decrease of external damping $(\mu=0.05)$ on the frequency response of the system. The nature of the shape of the curves is similar to that of Figure 4, but the jump phenomena occur at a higher value of parametric excitation frequency detuning parameter $\left(\sigma_{2}=382.9953\right)$, and the amplitudes of both directly and indirectly excited modes are amplified.

5.2. Dynamic Solutions. Frequency response and amplitude response plots reveal different stability and bifurcations of the 


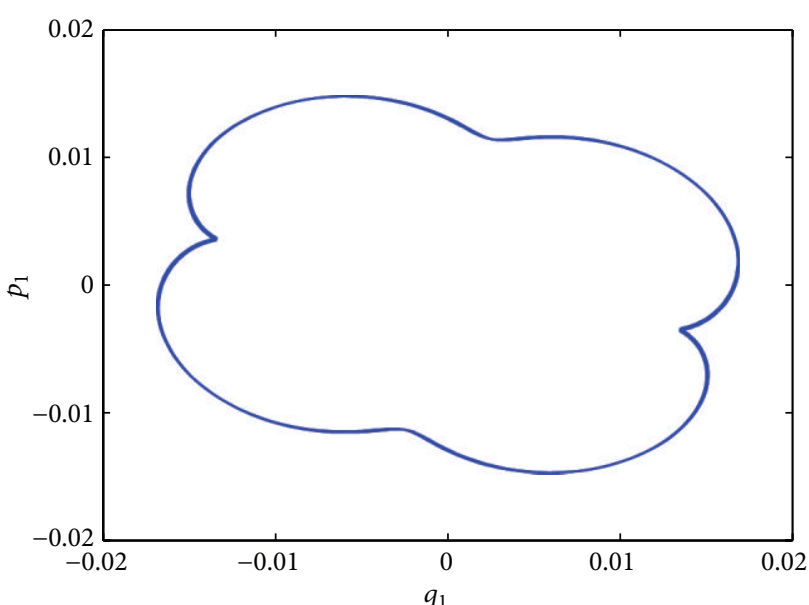

(a)

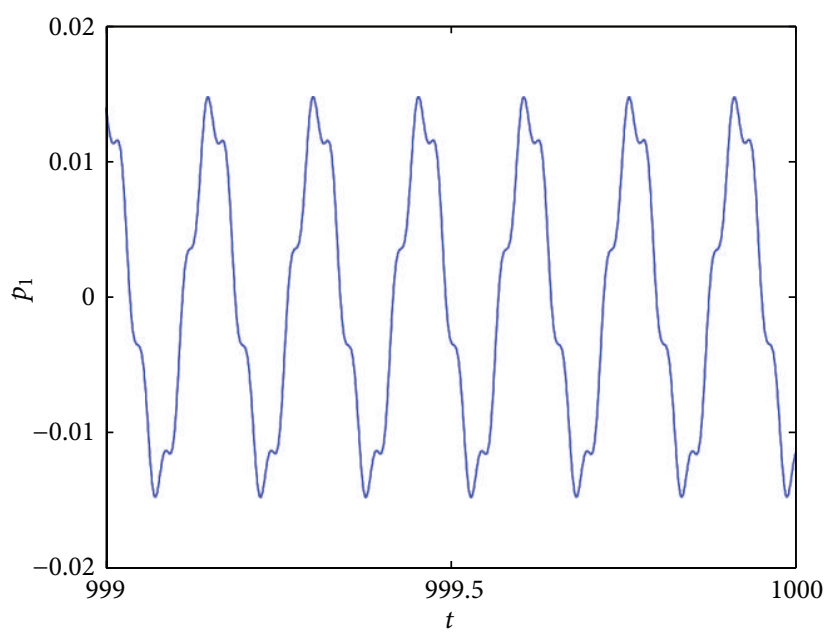

(c)

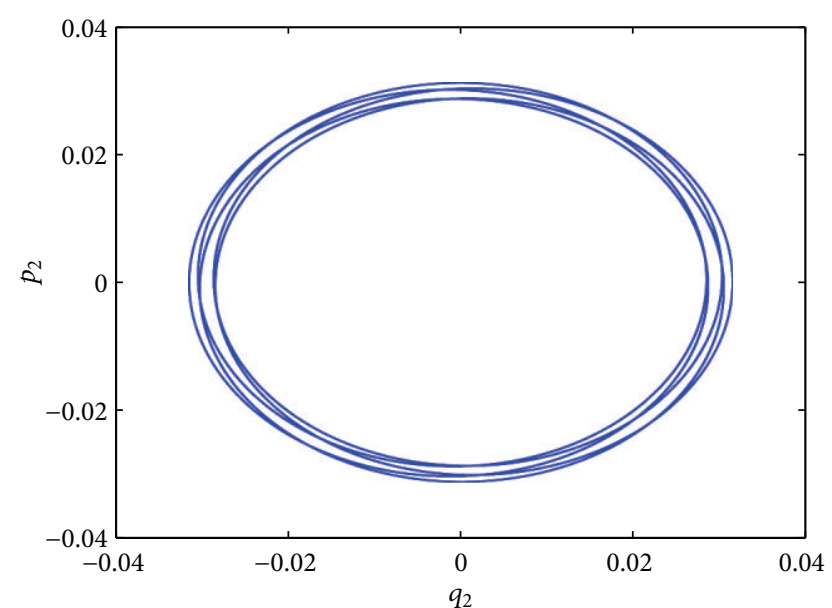

(b)

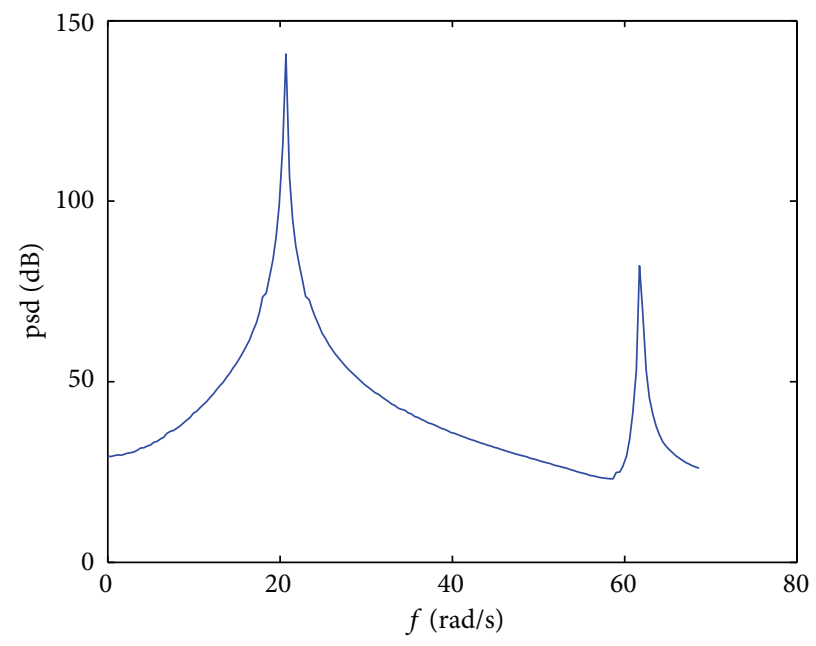

(d)

FIGURE 16: Phase portraits $(\mathrm{a}, \mathrm{b})$, time trace $(\mathrm{c})$, and FFT power spectra $(\mathrm{d})$ in the lower nontrivial stable branch of the frequency response plot of Figure 8 for $\sigma_{2}=280.5201, \mu=0.05, v_{1}=10, \alpha=0$, and $\sigma_{1}=92.39$.

equilibrium solutions with variation of control parameters. Dynamic analysis of the system which is dependent on initial conditions is studied in the form of periodic, quasiperiodic, and chaotic responses, and some selected results are presented.

Figures 9(a)-9(d) show typical system response in terms of phase portraits (a,b) and time traces (c, d) at $\sigma_{2}=68.2799$ corresponding to the upper nontrivial stable branch of the frequency response curve (Figure 8) for $\mu=0.05, \alpha=0$, $v_{1}=10$, and $\sigma_{1}=92.39$. The response is periodic about the nontrivial equilibrium solution when the time integration is started with the initial values $p_{1}=0.002, q_{1}=0.0007$, $p_{2}=0.0067$, and $q_{2}=0.0001$. Further along the same branch at $\sigma_{2}=75.2799$, the response is quasiperiodic in both modes being more prominent in the second mode as shown in the two-dimensional projections of the phase portraits onto the $p-q$ planes in Figures 12(a) and 12(b) and the time traces in Figures $10(\mathrm{c})$ and $10(\mathrm{~d})$. The response remains quasiperiodic in both modes for higher frequency detuning parameter values, typically at $\sigma_{2}=76.2799$ as shown in the closed loop Poincare maps and FFT power spectra in Figures 11(c), 11(d), and 11(b), respectively. With further increase in the value of detuning parameter, typically at $\sigma_{2}=78.2799$, we find the closed loops of Poincare map get merged and give a way to chaotic response in second mode in Figure 12(d). However, in first mode the system response is still quasiperiodic as seen from phase portrait (Figure 12(a)) and Poincare map (Figure 12(c)), respectively.

Figures 13(a)-13(d) show the typical system behavior at $\sigma_{2}=115.7201, \mu=0.05, \alpha=0, v_{1}=10$, and $\sigma_{1}=92.39$ in terms of phase portraits and time traces in Figures 13(a), 13(b), 13(c), and 13(d), respectively. The response is initially chaotic and jumps to the nearby stable trivial attractor. With further increase in detuning parameter, the system behavior changes drastically as shown in Figures 14(a)-14(d), at $\sigma_{2}=$ 116.4201 illustrating quasiperiodic motion in the first mode and chaotic motion in second mode. The dynamic response in both modes becomes chaotic at detuning parameter 
TABLE 1: Variation of natural frequencies for the first two modes with mean travelling velocity $v_{0}$ showing $3: 1$ internal resonance for $v_{f}=$ 0.2 .

\begin{tabular}{lccccc}
\hline$v_{0}$ & $\omega_{1}$ & $\omega_{2}$ & $3 \omega_{1}$ & $\varepsilon \sigma_{1}=\omega_{2}-3 \omega_{1}$ & $\left(\varepsilon \sigma_{1} / \omega_{1}\right) \%$ \\
\hline 0.4500 & 3.3149 & 9.7032 & 9.9447 & -0.2415 & -7.2853 \\
0.4600 & 3.2969 & 9.6857 & 9.8907 & -0.2050 & -6.2180 \\
0.4700 & 3.2786 & 9.6677 & 9.8358 & -0.1681 & -5.1272 \\
0.4800 & 3.2599 & 9.6493 & 9.7797 & -0.1304 & -4.0001 \\
0.4900 & 3.2407 & 9.6305 & 9.7221 & -0.0916 & -2.8265 \\
0.5000 & 3.2211 & 9.6113 & 9.6633 & -0.0520 & -1.6144 \\
0.5100 & 3.2011 & 9.5917 & 9.6033 & -0.0116 & -0.3624 \\
0.5110 & 3.1991 & 9.5897 & 9.5973 & -0.0076 & -0.2376 \\
0.5120 & 3.1970 & 9.5878 & 9.5910 & -0.0032 & -0.1001 \\
$\mathbf{0 . 5 1 3 0}$ & 3.1950 & $\mathbf{9 . 5 8 5 8}$ & $\mathbf{9 . 5 8 5 0}$ & $\mathbf{0 . 0 0 0 8}$ & $\mathbf{0 . 0 2 5 0}$ \\
0.5140 & 3.1930 & 9.5838 & 9.5790 & 0.0048 & 0.1503 \\
0.5600 & 3.0948 & 9.4876 & 9.2844 & 0.2032 & 6.5659 \\
0.5800 & 3.0493 & 9.4431 & 9.1479 & 0.2952 & 9.6809 \\
0.6000 & 3.0020 & 9.3968 & 9.0060 & 0.3908 & 13.0180 \\
\hline
\end{tabular}

$\sigma_{2}=117.0201$ as shown in phase portrait, FFT power spectra, and Poincare maps in Figures 15(a), 15(b), 15(c), and 15(d), respectively. The changes in system response from periodic in both modes to mixed mode, that is, quasiperiodic in first and chaotic in second mode to chaotic in both modes, as explained above, happen in the zone of frequency response plot where all three kinds of curves stable, saddle, and unstable are in very close proximity and crossing each other. Existence of multiple branches is possible due to the presence of internal resonance in the system. The nonlinear modal interaction influences simultaneously both the stable and unstable attractors, which finally results in such varied system responses.

Similar investigation is carried in the lower nontrivial stable branch of the frequency response plot of Figure 8 as well. For a point on the same branch at $\sigma_{2}=280.5201$, the system response exhibits one periodic and one quasiperiodic system behavior as shown in Figure 16 in terms of phase portraits, time trace, and FFT power spectra. The same behavior is noticed for another point on the same branch at $\sigma_{2}=310.5201$ though the figures are not presented to avoid repetition. Thus, due to the presence of internal resonance, a wide range of dynamic behavior can be observed with variation of control parameters.

\section{Conclusions}

In the present investigation, principal parametric resonance of first mode in presence of 3:1 internal resonance of a beam moving with variable velocity is considered. Stability boundaries of trivial state are obtained for different values of internal and external dissipations. It has been observed that higher values of damping have the effect of raising and narrowing the instability zones. Bifurcations of equilibrium solutions are analyzed in the form response plots. It has been shown in frequency response plot that the nontrivial steady state solutions bifurcate from trivial solutions through supercritical pitchfork bifurcations.

Besides the pitchfork bifurcations, the system also experiences Hopf bifurcation and saddle node bifurcation due to variation of different system parameters. Damping decreases the strength of nonlinear interaction due to internal resonance. Increasing amplitude of fluctuating velocity component broadens the range of trivial state instability and increases the value of parametric frequency detuning at which jump phenomena occur. Decreasing internal frequency detuning parameter affects the amplitude of directly excited first mode and number of Hopf bifurcation points. It also shifts the occurrence of jump phenomena.

A detailed study is carried out to determine the influence of different control parameters on dynamic behavior of the system. The dynamic solutions in the periodic, quasiperiodic, and chaotic forms, are captured with the help of time history, phase portraits, and Poincare maps. A wide array of dynamic behavior is noticed when nontrivial stable and saddle branches are formed due to internal resonance and also in the zone where the three branches are very close and crossing each other.

In case of conventional nontravelling beams with simply supported boundary conditions, occurrence of internal resonance is not possible due to vanishing of the nonlinear interaction coefficients [10]. However, a varied system response is possible in case of travelling beams due to nonlinear modal interaction leading to simultaneous influence of both stable and unstable attractors.

\section{Appendix}

We have the following:

$$
\begin{aligned}
& \Gamma_{1}=-2 i \omega_{1} A_{1}^{\prime} \phi_{1}-2 v_{0} A_{1}^{\prime} \phi_{1}^{\prime}-2 i \mu \omega_{1} A_{1} \phi_{1} \\
&-2 i \alpha \omega_{1} A_{1} \phi_{1}^{\prime \prime \prime \prime}+\frac{1}{2} v_{l}^{2} \\
& \times\left\{2 A_{1}^{2} \bar{A}_{1} \phi_{1}^{\prime \prime} \int_{0}^{1} \phi_{1}^{\prime} \bar{\phi}_{1}^{\prime} d x+A_{1}^{2} \bar{A}_{1} \bar{\phi}_{1}^{\prime \prime}\right. \\
& \times \int_{0}^{1} \phi_{1}^{\prime 2} d x+2 A_{1} A_{2} \bar{A}_{2} \bar{\phi}_{2}^{\prime \prime} \\
& \times \int_{0}^{1} \phi_{1}^{\prime} \phi_{2}^{\prime} d x+2 A_{1} A_{2} \bar{A}_{2} \phi_{1}^{\prime \prime} \\
&\left.\times \int_{0}^{1} \phi_{2}^{\prime} \bar{\phi}_{2}^{\prime} d x+2 A_{1} A_{2} \bar{A}_{2} \phi_{2}^{\prime \prime} \int_{0}^{1} \phi_{1}^{\prime} \bar{\phi}_{2}^{\prime} d x\right\}, \\
& \Gamma_{2}= \frac{1}{2} v_{l}^{2}\left\{2 \bar{A}_{1}^{2} A_{2} \bar{\phi}_{1}^{\prime \prime} \int_{0}^{1} \phi_{2}^{\prime} \bar{\phi}_{1}^{\prime} d x\right. \\
& \Gamma_{3}= \bar{A}_{1}\left\{v_{1} \omega_{1} \bar{\phi}_{1}^{\prime}-\frac{v_{1} \Omega}{2} \bar{\phi}_{1}^{\prime}+i v_{0} v_{1} \bar{\phi}_{1}^{\prime \prime}\right\}, \\
&\left.+\bar{A}_{1}^{2} A_{2} \phi_{2}^{\prime \prime} \int_{0}^{1} \bar{\phi}_{1}^{\prime 2} d x\right\},
\end{aligned}
$$


TABLE 2: Typical points on the different branches of the frequency response for $\mu=0.1, \alpha=0, v_{1}=10, v_{1}=40$, and $\sigma_{1}=92.39$.

\begin{tabular}{|c|c|c|c|c|c|}
\hline$p_{1}$ & $q_{1}$ & $p_{2}$ & $q_{2}$ & $\sigma_{2}$ & Bifurcation point \\
\hline 0.00500579 & -0.01569813 & 0.00004925 & 0.01964672 & 100 & \\
\hline-0.02181265 & -0.00742674 & 0.01533298 & 0.00020989 & 100 & \\
\hline 0.010164208 & 0.00356793 & 0.01833426 & 0.00011845 & 100 & \\
\hline-0.004819409 & 0.01479703 & 0.00009386 & 0.01071881 & 100 & \\
\hline-0.007939 & -0.003674 & 0.042869 & 0.006271 & 206.588482 & $H_{1}$ \\
\hline-0.002453 & -0.004177 & 0.039646 & 0.039917 & 301.873000 & SN \\
\hline 0.032196 & 0.010862 & 0.005854 & 0.000026 & 190.033742 & $\mathrm{H}_{2}$ \\
\hline 0.026628 & 0.008991 & 0.010665 & 0.000036 & 144.502843 & $\mathrm{H}_{3}$ \\
\hline 0.022130 & 0.007495 & 0.015112 & 0.000039 & 127.980336 & $H_{4}$ \\
\hline 0.004908 & 0.001822 & 0.013291 & 0.000245 & 80.408029 & $\mathrm{H}_{5}$ \\
\hline 0.000000 & 0.000000 & 0.000000 & 0.000000 & 49.474028 & $H_{6}$ \\
\hline 0.000000 & 0.000000 & 0.000000 & 0.000000 & 44.608834 & $\mathrm{H}_{7}$ \\
\hline
\end{tabular}

$$
\begin{aligned}
& \Gamma_{4}=A_{2}\left\{v_{1} \omega_{2} \phi_{2}^{\prime}-\frac{v_{1} \Omega}{2} \phi_{2}^{\prime}-i v_{0} v_{1} \phi_{2}^{\prime \prime}\right\}, \\
& \Gamma_{5}=-2 i \omega_{2} A_{2}^{\prime} \phi_{2}-2 v_{0} A_{2}^{\prime} \phi_{2}^{\prime}-2 \mu i \omega_{2} A_{2} \phi_{2} \\
& -2 \alpha i \omega_{2} A_{2} \phi_{2}^{\prime \prime \prime \prime}+\frac{1}{2} v_{l}^{2} \\
& \times\left\{A_{2}^{2} \bar{A}_{2} \bar{\phi}_{2}^{\prime \prime} \int_{0}^{1} \phi_{2}^{\prime 2} d x\right. \\
& +2 A_{1} \bar{A}_{1} A_{2} \phi_{2}^{\prime \prime} \int_{0}^{1} \phi_{1}^{\prime} \bar{\phi}_{1}^{\prime} d x+2 A_{1} \bar{A}_{1} A_{2} \bar{\phi}_{1}^{\prime \prime} \\
& \times \int_{0}^{1} \phi_{1}^{\prime} \phi_{2}^{\prime} d x+2 A_{2}^{2} \bar{A}_{2} \phi_{2}^{\prime \prime} \\
& \left.\times \int_{0}^{1} \phi_{2}^{\prime} \bar{\phi}_{2}^{\prime} d x+2 A_{1} \bar{A}_{1} A_{2} \phi_{1}^{\prime \prime} \int_{0}^{1} \phi_{2}^{\prime} \bar{\phi}_{1}^{\prime} d x\right\}, \\
& \Gamma_{6}=\frac{1}{2} v_{l}^{2}\left\{A_{1}^{3} \phi_{1}^{\prime \prime} \int_{0}^{1} \phi_{1}^{\prime 2} d x\right\}, \\
& \Gamma_{7}=A_{1}\left\{-v_{1} \omega_{1} \phi_{1}^{\prime}-\frac{v_{1} \Omega}{2} \phi_{1}^{\prime}+i v_{0} v_{1} \phi_{1}^{\prime \prime}\right\}, \\
& S_{1}=\left(\frac { 1 } { 1 6 } v _ { l } ^ { 2 } \left\{2 \int_{0}^{1} \phi_{1}^{\prime \prime} \bar{\phi}_{1} d x \int_{0}^{1} \phi_{1}^{\prime} \bar{\phi}_{1}^{\prime} d x\right.\right. \\
& \left.\left.+\int_{0}^{1} \bar{\phi}_{1}^{\prime \prime} \bar{\phi}_{1} d x \int_{0}^{1} \phi_{1}^{\prime 2} d x\right\}\right) \\
& \times\left(-\left\{i \omega_{1} \int_{0}^{1} \phi_{1} \bar{\phi}_{1} d x+v_{0} \int_{0}^{1} \phi_{1}^{\prime} \bar{\phi}_{1} d x\right\}\right)^{-1}, \\
& S_{2}=\left(\frac { 1 } { 8 } v _ { l } ^ { 2 } \left\{\int_{0}^{1} \bar{\phi}_{2}^{\prime \prime} \bar{\phi}_{1} d x \int_{0}^{1} \phi_{1}^{\prime} \phi_{2}^{\prime} d x+\int_{0}^{1} \phi_{1}^{\prime \prime} \bar{\phi}_{1} d x\right.\right. \\
& \left.\left.\times \int_{0}^{1} \phi_{2}^{\prime} \bar{\phi}_{2}^{\prime} d x+\int_{0}^{1} \phi_{2}^{\prime \prime} \bar{\phi}_{1} d x \int_{0}^{1} \phi_{1}^{\prime} \bar{\phi}_{2}^{\prime} d x\right\}\right) \\
& \times\left(-\left\{i \omega_{1} \int_{0}^{1} \phi_{1} \bar{\phi}_{1} d x+v_{0} \int_{0}^{1} \phi_{1}^{\prime} \bar{\phi}_{1} d x\right\}\right)^{-1},
\end{aligned}
$$

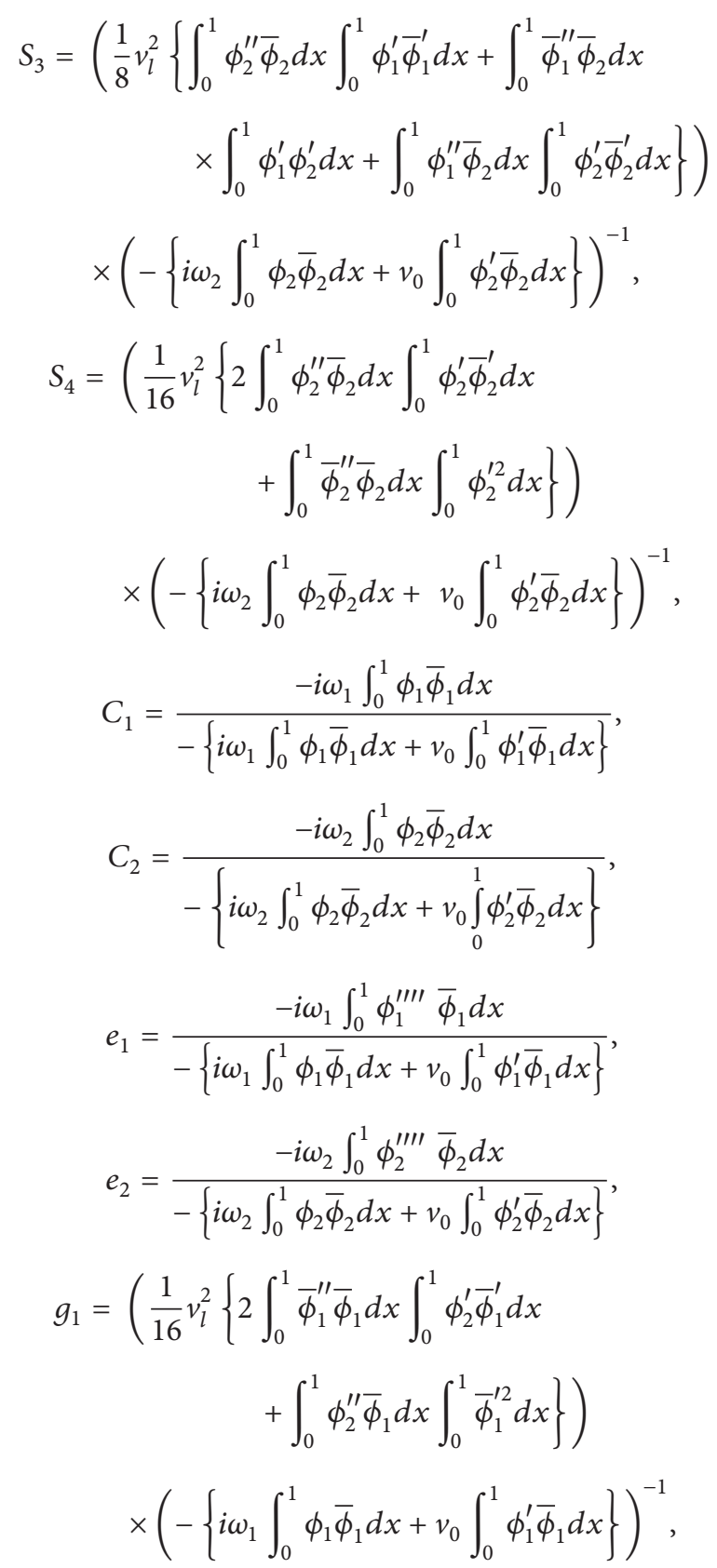




$$
\begin{gathered}
g_{2}=\frac{(1 / 16) v_{l}^{2}\left\{\int_{0}^{1} \phi_{1}^{\prime \prime} \bar{\phi}_{2} d x \int_{0}^{1} \phi_{1}^{\prime 2} d x\right\}}{-\left\{i \omega_{2} \int_{0}^{1} \phi_{2} \bar{\phi}_{2} d x+v_{0} \int_{0}^{1} \phi_{2}^{\prime} \bar{\phi}_{2} d x\right\}}, \\
K_{1}=\left(\frac { 1 } { 2 } \left\{v_{1} \omega_{1} \int_{0}^{1} \bar{\phi}_{1}^{\prime} \bar{\phi}_{1} d x-\frac{v_{1} \Omega}{2} \int_{0}^{1} \bar{\phi}_{1}^{\prime} \bar{\phi}_{1} d x\right.\right. \\
\left.\left.+i v_{0} v_{1} \int_{0}^{1} \bar{\phi}_{1}^{\prime \prime} \bar{\phi}_{1} d x\right\}\right) \\
\times\left(-\left\{i \omega_{1} \int_{0}^{1} \phi_{1} \bar{\phi}_{1} d x+v_{0} \int_{0}^{1} \phi_{1}^{\prime} \bar{\phi}_{1} d x\right\}\right)^{-1}, \\
K_{2}=\left(\frac { 1 } { 2 } \left\{v_{1} \omega_{2} \int_{0}^{1} \phi_{2}^{\prime} \bar{\phi}_{1} d x-\frac{v_{1} \Omega}{2} \int_{0}^{1} \phi_{2}^{\prime} \bar{\phi}_{1} d x\right.\right. \\
\left.\left.\quad-i v_{0} v_{1} \int_{0}^{1} \phi_{2}^{\prime \prime} \bar{\phi}_{1} d x\right\}\right) \\
\times\left(-\left\{i \omega_{1} \int_{0}^{1} \phi_{1} \bar{\phi}_{1} d x+v_{0} \int_{0}^{1} \phi_{1}^{\prime} \bar{\phi}_{1} d x\right\}\right)^{-1}, \\
K_{3}=\left(\frac { 1 } { 2 } \left\{-v_{1} \omega_{2} \int_{0}^{1} \phi_{1}^{\prime} \bar{\phi}_{2} d x-\frac{v_{1} \Omega}{2} \int_{0}^{1} \phi_{1}^{\prime} \bar{\phi} d x\right.\right. \\
\left.\times\left(-i v_{0} v_{1} \int_{0}^{1} \phi_{1}^{\prime \prime} \bar{\phi}_{2} d x\right\}\right) \\
\left.\times\left\{i \omega_{2} \int_{0}^{1} \phi_{2} \bar{\phi}_{2} d x+v_{0} \int_{0}^{1} \phi_{2}^{\prime} \bar{\phi}_{2} d x\right\}\right)^{-1} .
\end{gathered}
$$

\section{References}

[1] J. A. Wickert and C. D. Mote Jr., "Current research on the vibration and stability of axially moving materials," Shock and Vibration Digest, vol. 20, pp. 3-13, 1988.

[2] C. D. Mote Jr., "On the nonlinear oscillation of an axially moving string," Journal of Applied Mechanics, vol. 33, pp. 463-464, 1966.

[3] J. A. Wickert and C. D. Mote Jr., "Classical vibration analysis of axially moving continua," Journal of Applied Mechanics, vol. 57, no. 3, pp. 738-744, 1990.

[4] J. A. Wickert and C. D. Mote Jr., "Travelling load response of an axially moving string," Journal of Sound and Vibration, vol. 149, no. 2, pp. 267-284, 1991.

[5] J. A. Wickert, "Non-linear vibration of a traveling tensioned beam," International Journal of Non-Linear Mechanics, vol. 27, no. 3, pp. 503-517, 1992.

[6] G. Chakraborty, A. K. Mallik, and H. Hatwal, "Non-linear vibration of a travelling beam," International Journal of NonLinear Mechanics, vol. 34, no. 4, pp. 655-670, 1999.

[7] G. Chakraborty and A. K. Mallik, "Non-linear vibration of a travelling beam having an intermediate guide," Nonlinear Dynamics, vol. 20, no. 3, pp. 247-265, 1999.

[8] H. R. Öz and M. Pakdemirli, "Vibrations of an axially moving beam with time-dependent velocity," Journal of Sound and Vibration, vol. 227, no. 2, pp. 239-257, 1999.

[9] H. R. Öz, M. Pakdemirli, and H. Boyaci, "Non-linear vibrations and stability of an axially moving beam with time-dependent velocity," International Journal of Non-Linear Mechanics, vol. 36, no. 1, pp. 107-115, 2001.

[10] A. H. Nayfeh and D. T. Mook, Nonlinear Oscillations, Wiley, New York, NY, USA, 1979.

[11] A. H. Nayfeh and B. Balachandran, Applied Nonlinear Dynamics-Analytical, Computational and Experimental Methods, John Wiley \& Sons, New York, NY, USA, 1994.

[12] A. H. Nayfeh and B. Balachandran, "Modal interactions in dynamical and structural systems," Applied Mechanics Reviews, vol. 42, pp. 175-201, 1989.

[13] C. H. Riedel and C. A. Tan, "Coupled, forced response of an axially moving strip with internal resonance," International Journal of Non-Linear Mechanics, vol. 37, no. 1, pp. 101-116, 2002.

[14] E. Özkaya, S. M. Bağdatli, and H. R. Öz, "Nonlinear transverse vibrations and 3:1 internal resonances of a beam with multiple supports," Journal of Vibration and Acoustics, vol. 130, no. 2, Article ID 021013, 11 pages, 2008.

[15] S. M. Bağdatli, H. R. Öz, and E. Özkaya, "Non-linear transverse vibrations and $3: 1$ internal resonances of a tensioned beam on multiple supports," Mathematical and Computational Applications, vol. 16, no. 1, pp. 203-215, 2011.

[16] C. Chin and A. H. Nayfeh, "Three-to-one internal resonances in parametrically excited hinged-clamped beams," Nonlinear Dynamics, vol. 20, no. 2, pp. 131-158, 1999.

[17] L. N. Panda and R. C. Kar, "Nonlinear dynamics of a pipe conveying pulsating fluid with parametric and internal resonances," Nonlinear Dynamics, vol. 49, no. 1-2, pp. 9-30, 2007.

[18] L. N. Panda and R. C. Kar, "Nonlinear dynamics of a pipe conveying pulsating fluid with combination, principal parametric and internal resonances," Journal of Sound and Vibration, vol. 309, no. 3-5, pp. 375-406, 2008.

[19] K. Y. Sze, S. H. Chen, and J. L. Huang, "The incremental harmonic balance method for nonlinear vibration of axially moving beams," Journal of Sound and Vibration, vol. 281, no. 35, pp. 611-626, 2005.

[20] J. L. Huang, R. K. L. Su, W. H. Li, and S. H. Chen, "Stability and bifurcation of an axially moving beam tuned to three-toone internal resonances," Journal of Sound and Vibration, vol. 330, no. 3, pp. 471-485, 2011.

[21] L. Chen, Y. Tang, and C. W. Lim, "Dynamic stability in parametric resonance of axially accelerating viscoelastic Timoshenko beams," Journal of Sound and Vibration, vol. 329, no. 5, pp. 547$565,2010$.

[22] H. Ding and L. Chen, "Galerkin methods for natural frequencies of high-speed axially moving beams," Journal of Sound and Vibration, vol. 329, no. 17, pp. 3484-3494, 2010.

[23] H. Ding, G. C. Zhang, and L. Q. Chen, "Supercritical equilibrium solutions of axially moving beams with hybrid boundary conditions," Mechanics Research Communications, vol. 38, no. 1, pp. 52-56, 2011.

[24] K. Marynowski and T. Kapitaniak, "Kelvin-Voigt versus Bürgers internal damping in modeling of axially moving viscoelastic web," International Journal of Non-Linear Mechanics, vol. 37, no. 7, pp. 1147-1161, 2002.

[25] K. Marynowski, "Non-linear vibrations of an axially moving viscoelastic web with time-dependent tension," Chaos, Solitons and Fractals, vol. 21, no. 2, pp. 481-490, 2004.

[26] K. Marynowski and T. Kapitaniak, "Zener internal damping in modelling of axially moving viscoelastic beam with time-dependent tension," International Journal of Non-Linear Mechanics, vol. 42, no. 1, pp. 118-131, 2007. 
[27] M. Pakdemirli and H. R. Öz, "Infinite mode analysis and truncation to resonant modes of axially accelerated beam vibrations," Journal of Sound and Vibration, vol. 311, no. 3-5, pp. 1052-1074, 2008

[28] S. V. Ponomareva and W. T. van Horssen, "On the transversal vibrations of an axially moving continuum with a time-varying velocity: transient from string to beam behavior," Journal of Sound and Vibration, vol. 325, no. 4-5, pp. 959-973, 2009.

[29] M. H. Ghayesh, "Nonlinear forced dynamics of an axially moving viscoelastic beam with an internal resonance," International Journal of Mechanical Sciences, vol. 53, no. 11, pp. 1022-1037, 2011.

[30] M. H. Ghayesh, H. A. Kafiabad, and T. Reid, "Sub- and supercritical nonlinear dynamics of a harmonically excited axially moving beam," International Journal of Solids and Structures, vol. 49, no. 1, pp. 227-243, 2012.

[31] M. H. Ghayesh, "Coupled longitudinal-transverse dynamics of an axially accelerating beam," Journal of Sound and Vibration, vol. 331, pp. 5107-5124, 2012.

[32] M. H. Ghayesh, "Subharmonic dynamics of an axially accelerating beam," Archive of Applied Mechanics, vol. 82, pp. 1169-1181, 2012.

[33] M. H. Ghayesh and M. Amabili, "Steady-state transverse response of an axially moving beam with time-dependent axial speed," International Journal of Non-Linear Mechanics, vol. 49, pp. 40-49, 2013.

[34] G. Chakraborty and A. K. Mallik, "Stability of an accelerating beam," Journal of Sound and Vibration, vol. 27, no. 2, pp. 309320, 1999.

[35] M. P. Paidoussis, "Flutter of conservative systems of pipe conveying incompressible fluid," Journal of Mechanical Engineering and Science, vol. 17, no. 1, pp. 19-25, 1975.

[36] M. Pakdemirli and H. Boyaci, "Comparision of direct perturbation methods with discretization-perturbation methods for nonlinear vibrations," Journal of Sound and Vibration, vol. 186, pp. 837-845, 1985.

[37] A. H. Nayfeh and P. F. Pai, Linear and Nonlinear Structural Mechanics, Wiley-Interscience, New York, NY, USA, 2004. 

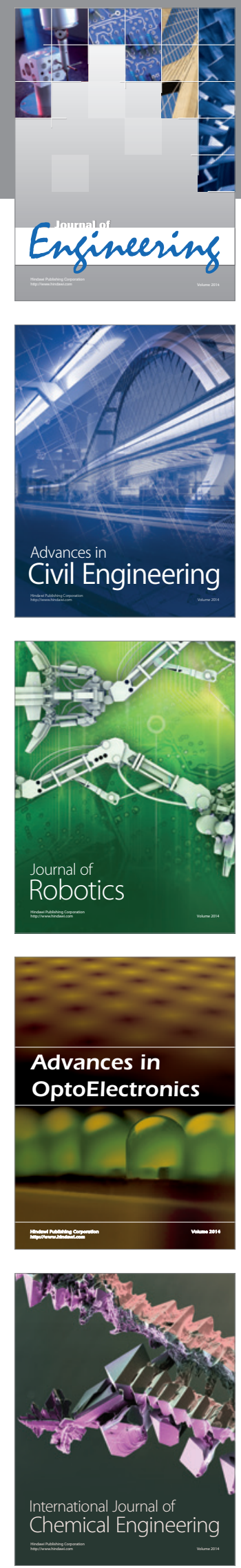

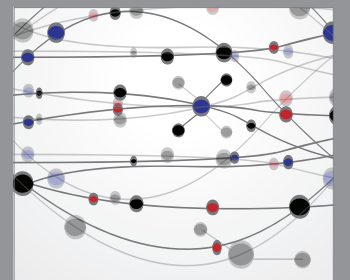

The Scientific World Journal
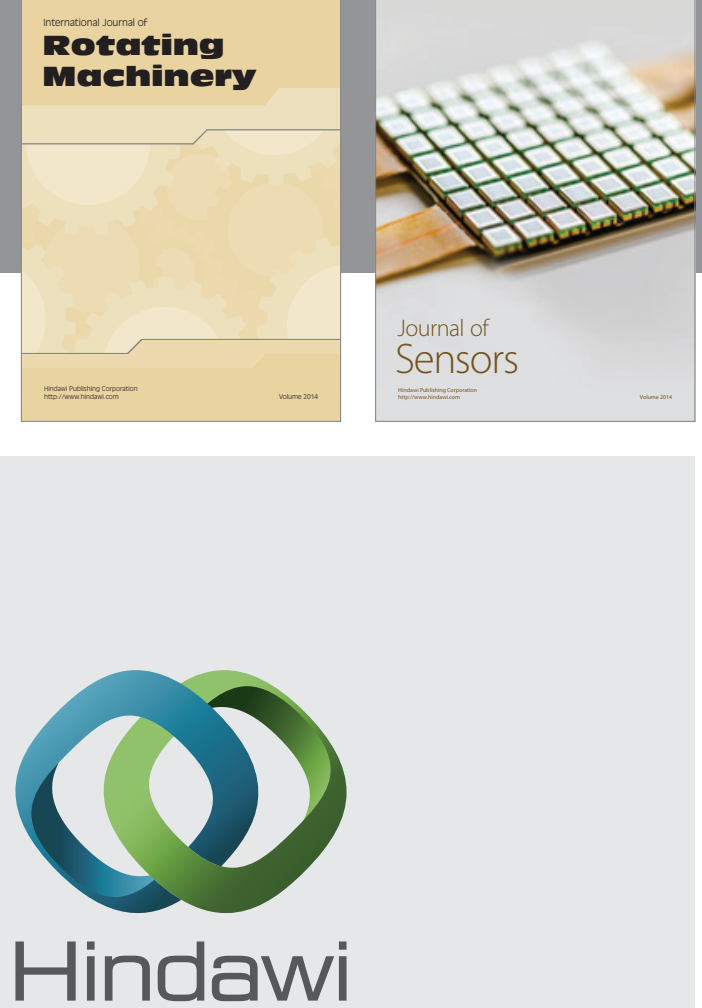

Submit your manuscripts at http://www.hindawi.com
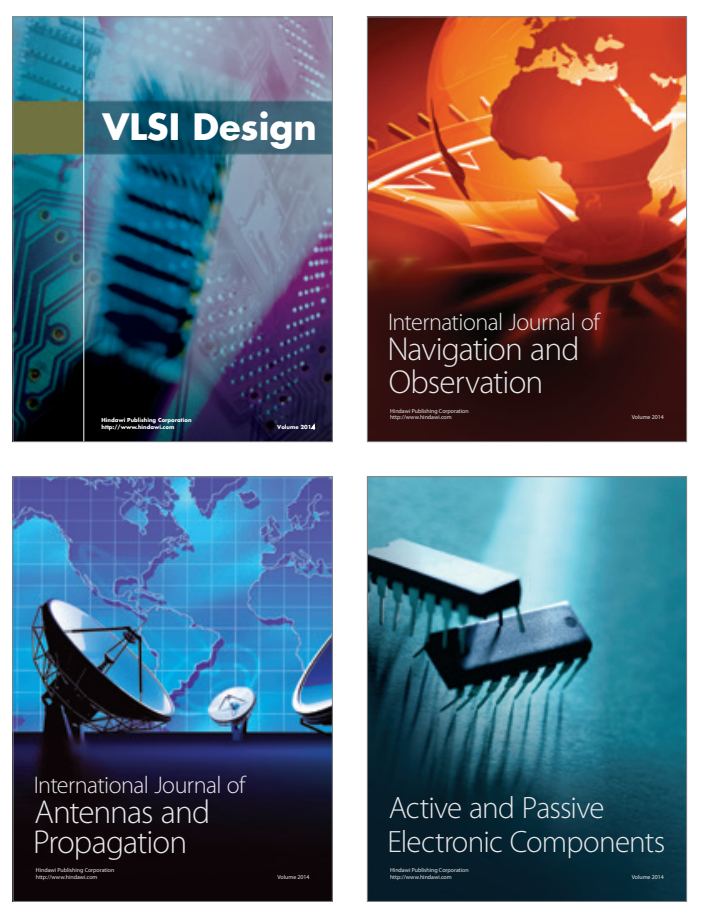
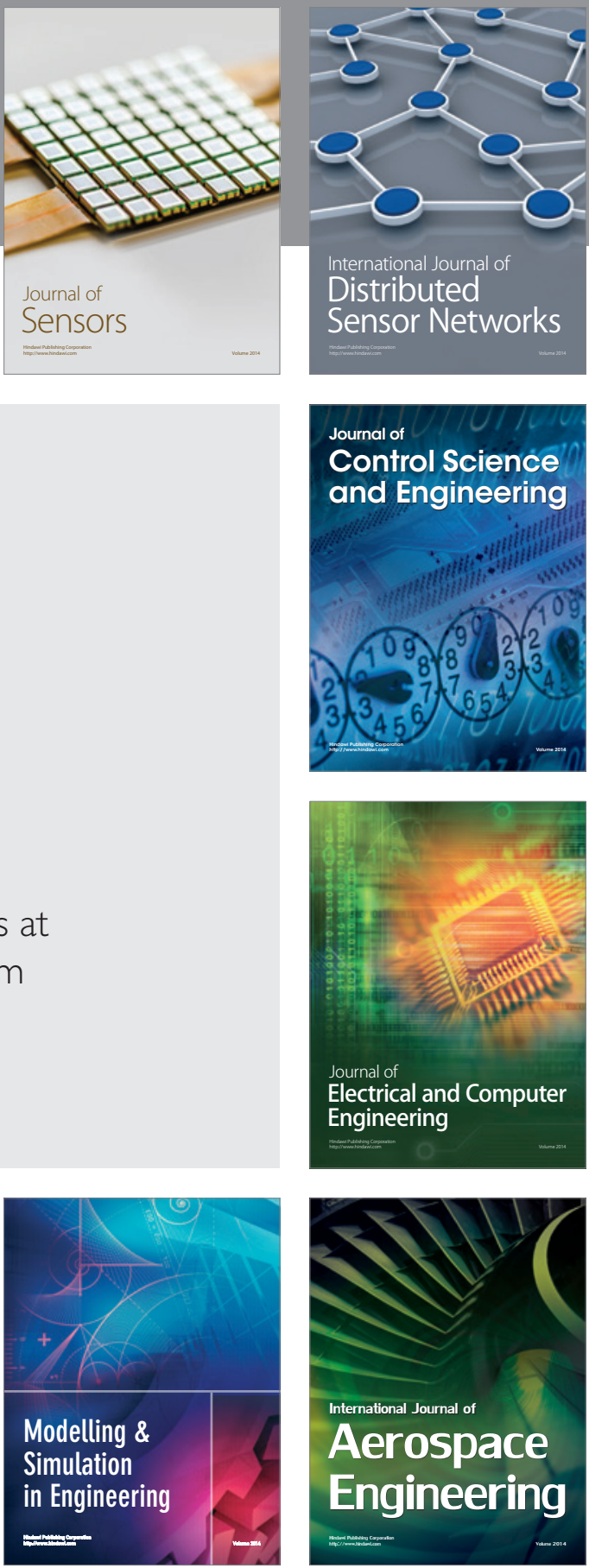

Journal of

Control Science

and Engineering
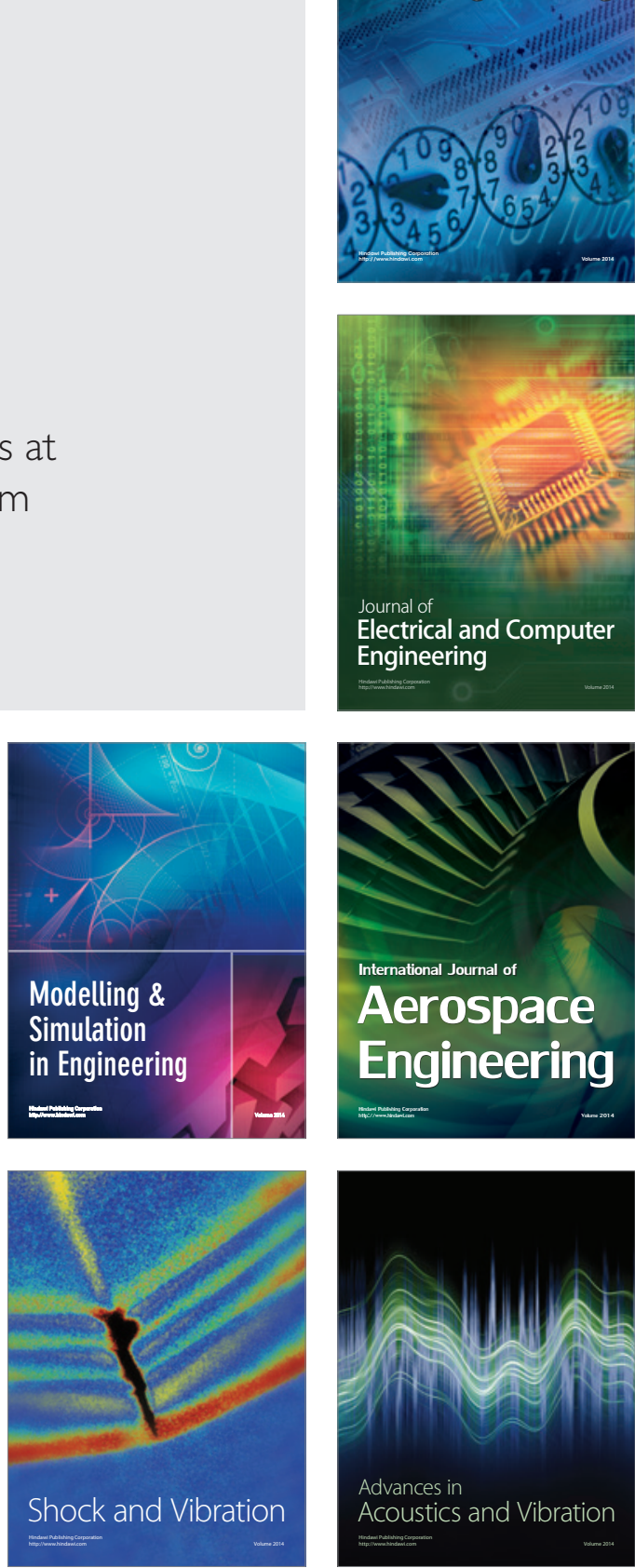